\title{
A Distributed Lag Analysis of the Relationship Between Gibberella zeae Inoculum Density on Wheat Spikes and Weather Variables
}

\author{
P. A. Paul, P. E. Lipps, E. De Wolf, G. Shaner, G. Buechley, T. Adhikari, \\ S. Ali, J. Stein, L. Osborne, and L. V. Madden
}

First, second, and tenth authors: Department of Plant Pathology, The Ohio State University, Ohio Agricultural Research and Development Center, Wooster 44691; third author: Department of Plant Pathology, Kansas State University, Manhattan 66506; fourth and fifth authors: Department of Botany and Plant Pathology, Purdue University, West Lafayette, IN 47907; sixth and seventh authors: Department of Plant Pathology, North Dakota State University, Fargo 58105; and eighth and ninth authors: Plant Science Department, South Dakota State University, Brookings 57007.

Accepted for publication 19 July 2007.

\begin{abstract}
Paul, P. A., Lipps, P. E., De Wolf, E., Shaner, G., Buechley, G., Adhikari, T., Ali, S., Stein, J., Osborne, L., and Madden, L. V. 2007. A distributed lag analysis of the relationship between Gibberella zeae inoculum density on wheat spikes and weather variables. Phytopathology 97:1608-1624.

In an effort to characterize the association between weather variables and inoculum of Gibberella zeae in wheat canopies, spikes were sampled and assayed for pathogen propagules from plots established in Indiana, North Dakota, Ohio, Pennsylvania, South Dakota, and Manitoba between 1999 and 2005. Inoculum abundance was quantified as the daily number of colony forming units per spike (CFU/spike). A total of 49 individual weather variables for $24-\mathrm{h}$ periods were generated from measurements of ambient weather data. Polynomial distributed lag regression analysis, followed by linear mixed model analysis, was used to (i) identify weather variables significantly related to log-transformed CFU/spike (the response

polynomial degree for the relationship between the parameter weights for the weather variables and the time lag involved), and (iv) account for location-specific effects and random effects of years within locations on the response variable. Both location and year within location affected the magnitude of $Y$, but there was no consistent trend in $Y$ over time. $Y$ on each day was significantly and simultaneously related to weather variables on the day of sampling and on the 8 days prior to sampling (giving a 9-day time window). The structural relationship corresponded to polynomial degrees of 0,1 , or 2 , generally showing a smooth change in the parameter weights and time lag. Moisture- (e.g., relative humidity-) related variables had the strongest relationship with $Y$, but air temperatureand rainfall-related variables also significantly affected $Y$. The overall marginal effect of each weather variable on $Y$ was positive. Thus, local weather conditions can be utilized to improve estimates of spore density on wheat spikes around the time of flowering.
\end{abstract} variable; $Y$ ), (ii) determine the time window (i.e., lag length) over which each weather variable affected $Y$, (iii) determine the form of the relationship between each weather variable and $Y$ (defined in terms of the

Fusarium head blight of wheat (Triticum aestivum L.) is caused predominantly by Gibberella zeae (Schwein.) Petch (anamorph: Fusarium graminearum Schwabe) in North America. The increased intensity of this disease over the past 15 years has been attributed to the widespread adoption of minimum tillage, a practice which favors the survival of $G$. zeae between growing seasons $(6,14,28,37)$. Tillage and crop rotation are management strategies often recommended to reduce residue-borne inoculum density and pathogen survival; however, for an individual field, these strategies may be ineffective because of the ingress of spores from external sources. Wind $(20,47)$ or rain splash $(45)$ disseminate spores produced in crop residue left on the soil surface to wheat spikes, causing severe blighting under favorable weather conditions. Fusarium head blight (FHB) develops best when wet, humid conditions occur during or near flowering, and disease intensity is generally greater when wheat follows host crops such as corn or wheat than when wheat follows nonhost crops such as soybean in a cropping sequence $(13,14,50,61)$. The number of viable propagules of $F$. graminearum $/ G$. zeae sampled

Corresponding author: L. V. Madden; E-mail address: madden.1@ osu.edu

doi:10.1094/PHYTO-97-12-1608

(c) 2007 The American Phytopathological Society
Additional keywords: Fusarium graminearum, Fusarium head blight, wheat scab.

in and around wheat fields generally is higher in fields with host crop residue than in those with nonhost crop residue $(23,50)$, and in fields located in epidemic areas than in nonepidemic areas (23).

Research suggests that there is an association between inoculum density or abundance and FHB development $(2,5,19)$, as well as deoxynivalenol (DON) accumulation in wheat grain (14, $50,51)$. DON is a mycotoxin produced by $F$. graminearum that poses a health threat to humans and livestock. Based on greenhouse inoculations of wheat spikes, Fauzi and Paulitz (19) reported that $\log$ spore concentration explained $91 \%$ of the variation in arcsine-transformed percentage of spikelet diseased. According to Bai (5), as the number of spores of G. zeae inoculated per floret increased, incubation period decreased and FHB incidence increased. Stein and Osborne (58) reported a significant, positive correlation between the presence of inoculum on wheat spikes during heading and DON content of harvested grain. In addition, reports of positive associations (under favorable weather conditions) among cropping sequence (wheat following corn), conservation tillage practices, host crop surface residue, and DON contamination $(14,50,51)$ provide circumstantial evidence of an association between inoculum abundance and DON accumulation. These findings clearly suggest that the abundance of inoculum of $F$. graminearum/G. zeae is an important risk factor for FHB development and DON accumulation. An understanding of the dynamic associations among inoculum density, toxin produc- 
tion, weather, and disease intensity would be invaluable for ongoing efforts to predict the risk of FHB and DON.

The deployment of airborne spore samplers or traps and assays of detached wheat spikes are the most commonly used methods of monitoring the abundance of propagules of $F$. graminearum/G. zeae within wheat fields and evaluating the association among inoculum density, FHB intensity, and weather conditions. Airborne spore sampling alone, however, may be misleading, because spores in the air are not always destined to reach and infect wheat spikes. Some airborne spores may be deposited onto leaves or the ground, or even be blown away by wind. There is not always a high correlation between spores collected in Burkhard airborne spore samplers and spores washed from wheat spikes $(12,41$, 44,57). Furthermore, the correlation between airborne spore counts and FHB intensity is often very weak (50). Assays of wheat spikes for propagules, on the other hand, give a more direct estimate of the amount of inoculum reaching the infection court. However, like airborne spore sampling, direct spike assays for propagules can be tedious, time-consuming, and difficult to implement as risk tools for practical and timely disease management decision-making. Both approaches require periodic collection and processing of samples, and characterization, identification, and enumeration of propagules and colonies. This generally takes several days or weeks.

A practical approach to using inoculum density as a risk factor for FHB and DON would be to estimate inoculum abundance based on the relationship between weather variables and spores sampled from wheat spikes. In order for spores to reach the spikes, they first have to be produced, released, transported, and deposited. Several weather variables influence these processes $(4,10,15,45,47-49,65,68)$. Based on our current understanding of the associations among environmental variables and $F$. graminearum/G. zeae spore production and dissemination, we proposed to investigate the relationship between the number of spores sampled from wheat spikes and weather variables, and develop models describing this relationship. More specifically, we used polynomial distributed lag (PDL) regression $(7,8,55)$ coupled with linear mixed model analysis $(24,33)$ to determine the relationship between weather variables and their lag distributions on inoculum density on wheat spike across multiple locations. This analysis represents a novel approach in plant pathology to relate weather data to pathogen inoculum in the field.

\section{MATERIALS AND METHODS}

Spike sampling and weather monitoring. Samples of wheat spikes were collected at multiple locations from 1999 to 2005 (Table 1) and assayed for propagules of G. zeae according to a standard protocol. Five spikes were collected daily at approximately 11:00 a.m. between Feekes GS 10 and 11.2 (31) from each of multiple (3 to 9) research plots. In most cases, each set of five spikes was washed in $50 \mathrm{ml}$ of sterile distilled water, however, on some occasions spikes were washed individually in $10 \mathrm{ml}$ of sterile distilled water. Aliquots of spike wash suspensions were transferred to (3 to 5) replicate plates of Komada's selective medium (29) and colonies were grown at room temperature for 7 to 10 days under a 12-h photoperiod. Colony forming units (CFUs) per plate were counted and categorized based on spore and colony morphology. In some instances, the spike wash suspension was diluted before being plated. Sample colonies of each type were transferred to potato dextrose agar (PDA), carrot agar, and/or carnation leaf agar for identification of Fusarium species (38). At each location, for each day of sampling, the average number of CFUs of $G$. zeae across replicate plates was converted to CFUs per spike based on the volume of water used to wash the spikes, the number of spikes washed, and the dilution factor.

An automated weather station (Campbell Scientific Inc., Model CR10X, Provo, UT) was deployed at each location to record tem-

TABLE 1. Sampling wheat spikes at multiple locations over multiple years for propagules of Gibberella zeae

\begin{tabular}{|c|c|c|c|c|}
\hline Location $^{\mathrm{a}}$ & Year & Days $^{b}$ & Period $^{c}$ & Mean $(\text { range })^{\mathrm{d}}$ \\
\hline \multirow[t]{3}{*}{ Indiana } & 2001 & 36 & $130-165$ & $92(0-900)$ \\
\hline & 2002 & 36 & $135-171$ & $101(1-750)$ \\
\hline & 2004 & 18 & $132-150$ & $37(1-124)$ \\
\hline Manitoba, Canada & 2001 & 21 & $200-222$ & $72(27-188)$ \\
\hline \multirow[t]{4}{*}{ North Dakota } & 1999 & 20 & $179-198$ & $10(0-59)$ \\
\hline & 2000 & 19 & $177-196$ & $31(0-128)$ \\
\hline & 2001 & 19 & $182-201$ & $16(0-138)$ \\
\hline & 2005 & 18 & $174-196$ & $53(12-136)$ \\
\hline \multirow[t]{6}{*}{ Ohio } & 1999 & 22 & $139-160$ & $3(0-16)$ \\
\hline & 2000 & 20 & $136-155$ & $10(0-97)$ \\
\hline & 2001 & 20 & $141-160$ & $25(3-126)$ \\
\hline & 2002 & 26 & $143-168$ & $53(7-209)$ \\
\hline & 2003 & 33 & $142-174$ & $130(12-694)$ \\
\hline & 2004 & 13 & $139-152$ & $17(4-47)$ \\
\hline \multirow[t]{5}{*}{ South Dakota } & 2000 & 24 & $171-195$ & $51(0-207)$ \\
\hline & 2001 & 27 & $183-211$ & $797(9-5,787)$ \\
\hline & 2003 & 14 & $174-192$ & $224(24-897)$ \\
\hline & 2004 & 24 & $175-201$ & $1,059(71-4,287)$ \\
\hline & 2005 & 19 & $171-191$ & $664(107-1,700)$ \\
\hline
\end{tabular}

${ }^{a}$ United States or Canadian province from which wheat spikes were sampled and assayed for propagules of G. zeae in order to model the relationship between spike inoculum density and weather.

${ }^{b}$ Numbers of days that samples were collected. On any given day, from three to nine samples of five spikes each were collected from multiple research plots planted with locally adapted wheat cultivars.

${ }^{c}$ Sampling period in day of the year.

${ }^{\mathrm{d}}$ Mean (averaged across all samples collected during a given year) and minimum and maximum (in parentheses) daily number of propagules recovered from wheat spikes colony forming units (CFU/spike). 
perature (degree Celsius), rainfall (millimeters), relative humidity (percentage), wind speed (meters per second), and wind direction (degrees) at 30-min intervals. Rainfall amounts were recorded using a Tipping Bucket Rain Gauge (Model TE525WS, Campbell Scientific Inc., Provo, UT) with a 20-cm-diameter collector and a resolution of $0.25 \mathrm{~mm}$ of rain per tip. In about half of the location-years, surface wetness also was measured (determined as the change in kilo-ohms of electrical resistance from the threshold value, converted to a nominal scale [dry or wet]). In general, two sets of temperature and relative humidity sensors and between three and six surface wetness sensors were deployed. With the exception of wind gauges (placed approximately $2.5 \mathrm{~m}$ above the wheat canopy), all sensors and rain gauges were placed at spike height (approximately $100 \mathrm{~cm}$ above the soil surface). Another set of temperature and relative humidity sensors was placed at approximately 150 to $200 \mathrm{~cm}$ above the canopy to monitor (ambient) conditions of the air.

The collected weather data were manipulated to generate for each day; (i) averages, maxima, and minima for each weather variable, (i) cumulative hours of surface wetness, and temperature and relative humidity within specified ranges, and (iii) amount, duration, and intensity (millimeters per hour) of rainfall. To avoid discontinuity in high relative humidity and surface wetness periods, a day was defined as the 24-h period beginning at $1200 \mathrm{~h}$ on 1 day and ending at $1200 \mathrm{~h}$ on the following day. For each 24-h period, the number of hours was determined with (i) temperature $>9^{\circ} \mathrm{C}$ and between 15 and $30^{\circ} \mathrm{C}$, (ii) relative humidity $>80$ and $>90 \%$, and (iii) coincidence of combinations of temperature and relative humidity within the aforementioned ranges. Weather variables were also summarized for 12 -h periods, generating daytime (0600 to $1800 \mathrm{~h})$ and nighttime (1800 to $0600 \mathrm{~h})$ summaries of temperature and relative humidity. For temperature and relative humidity, 12- and 24-h summaries were generated for both canopy and air measurements.

Data analysis approach. To characterize the relationship between the daily density of propagules of G. zeae sampled from wheat spikes (CFU/spike; Z) and weather variables, a two-stage approach was used. In the first stage, the structure of the relationship was determined for each weather variable under consideration. Composite weather variables were then constructed based on the results from the first stage. In the second stage, linear mixed model analysis was used to combine the composite weather variables in a single model and simultaneously account for serial (i.e., temporal) correlations of data within each location-year and variation among location-years. Each stage is described below. For all analyses, the response variable $(Y)$ was the natural log of the daily

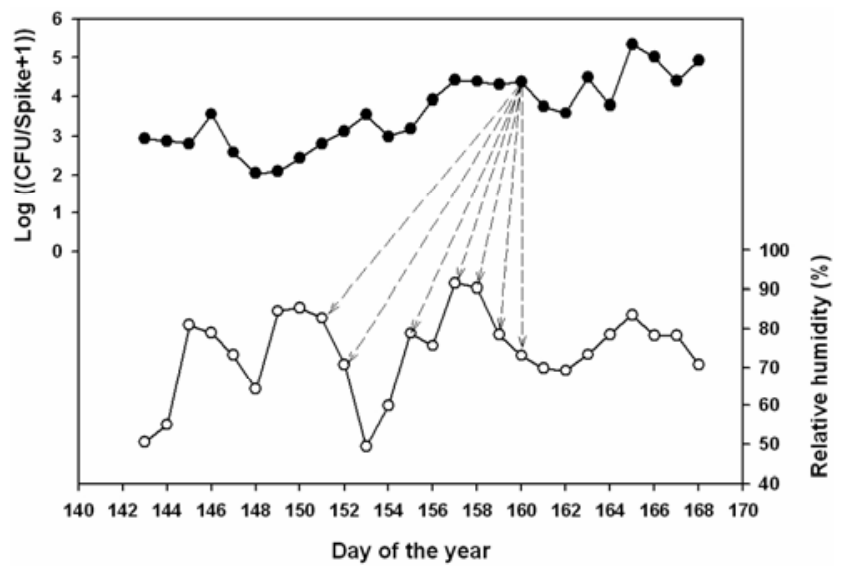

Fig. 1. Example of distributed-lag association between transformed daily spore density of Gibberella zeae on wheat spikes $[Y=\log (\mathrm{CFU} / \mathrm{spike}+1)]$ and relative humidity on the current day and on several days prior to the current day. The set of arrows originating at $Y$ on day 160 could be drawn from any of the observed $Y$ values.
CFU per spike plus one, that is, $Y=\log (Z+1)$. Transformation was done to stabilize variances and create a linear scale (52).

PDLs. A distributed-lag regression $(7,8,55)$ modeling approach was used in the first stage of the analysis to describe the relationship between $Y$ and weather variables. The conceptual model for this approach was built on the premise that the number of spores on the spike at any given time was a function of weather conditions favorable for spore production, maturation, liberation, dissemination, and deposition on the current day and for some period of time prior to the current day. This concept is demonstrated in Figure 1 for example profiles of $Y$ and one weather variable versus time of sampling. This concept is equivalent to the premise that weather conditions on any given day not only influence the number of spores on the spike on that day but also affect the numbers on several subsequent days (55). In a preliminary analysis of the relationship between the environment and $Y$, extended temporal windows of weather variables (summaries for consecutive 5- and 7-day periods prior to the day samples were collected) had stronger correlations with $Y$ than weather variables summarized for just the last $24 \mathrm{~h}$ prior to sampling (46). Similarly, Osborne and Stein (40) reported a lag association between various weather variables and the abundance of airborne spores of G. zeae. In another study, no significant relationship was found between G. zeae inoculum levels on wheat spikes and just coincident 24-h summaries of weather variables (35).

For a single weather variable $(X)$ and a single series of observations (i.e., for a single data set), the conceptual model can be written as

$$
Y_{t}=f\left(X_{t}, X_{t-1}, X_{t-2}, \ldots, X_{t-n}\right)+e_{t}
$$

where $Y_{t}$ is the response on day $t, e_{t}$ is the residual (error term), and $f(\bullet)$ is a function of $X$ on the current and previous days, up to $n$ days into the past. We only consider linear models in this study. The distributed lag (linear) model is written as

$$
Y_{t}=\alpha+\beta_{0} X_{t}+\beta_{1} X_{t-1}+\beta_{2} X_{t-2}+\ldots+\beta_{n} X_{t-n}+e_{t}
$$

where $\beta_{0}, \beta_{1}, \ldots, \beta_{n}$ are parameters corresponding to $X$ on the current day $\left(X_{t}\right.$; lag 0$)$, the previous day $\left(X_{t-1}\right.$; lag 1$)$, and earlier days, and $\alpha$ is the intercept parameter (expected $Y$ when all $X$ values are 0 ). The symbol $\beta_{m}$ is used to represents an individual $\beta$ at a specific lag $(m=0, \ldots, n)$. One can write the sum of the individual $\beta$ values as $\beta^{*}=\beta_{0}+\beta_{1}+\ldots+\beta_{n}=\Sigma \beta_{m}$. Then, the relative weight given to $X$ on each day (in terms of predicting $Y$ ) is specified by dividing the day-specific $\beta$ (e.g., $\beta_{1}$ ) by $\beta^{*}$ (e.g., $w_{1}=$ $\left.\beta_{1} / \beta^{*}, w_{m}=\beta_{m} / \beta^{*}\right)$. Thus, equation 1a can be written equivalently as

$$
Y_{t}=\alpha+\beta^{*}\left(w_{0} X_{t}+w_{1} X_{t-1}+w_{2} X_{t-2} \ldots+w_{n} X_{t-n}\right)+e_{t}
$$

The sum of the $w$ values equals 1 (i.e., $w_{0}+w_{1}+\ldots+w_{n}=1$ ). The composite $\beta^{*}$ parameter is the marginal effect of the weighted average weather variable on $Y$. Since the $w$ 's sum to $1, \beta^{*}$ represents the change in $Y$ with a unit change in $X$ (spread out over possibly several days). One can define the expression in parentheses in equation $1 \mathrm{~b}$ as $X_{t}^{*}$; then the model can be written simply as

$$
Y_{t}=\alpha+\beta^{*} X_{t}^{*}+e_{t}
$$

Because there is substantial correlation of weather variables over time, unconstrained estimates of the $\beta$ s will be imprecise and model fitting results unstable $(7,8,55,62)$. With a large temporal window under consideration (i.e., large $n$ ), there will be many separate $\beta$ s to estimate (one for each day), further adding to the instability of the estimated values. One approach to reduce the effect of correlated predictor variables is to constrain the values of the $\beta \mathrm{s}$; the approach taken here is to constrain the $\beta$ s to fit some polynomial function. This gives rise to a PDL regression model $(1,55,62)$. Explanation of the PDL methodology is given in the Appendix. 
Model fitting. Each daily transformed spore density value $(Y)$ and its corresponding vector of summarized weather variables at each location and year was treated as a separate observation, giving a total of 581 observations (118 from Indiana, 21 from Manitoba, 115 from North Dakota, 108 from South Dakota, 148 from Ohio, and 71 from Pennsylvania). The six locations represented a total of 27 location-years. The summarized weather variables for the 8 days prior to the first observation of $Y$ (with corresponding missing values for $Y$ ) were also included in the data set for each location-year, so that all observed $Y$ values could be utilized in the analysis. To account for overall location differences unrelated to weather conditions at and shortly before spore sampling, equation 1a was expanded to include a categorical location effect, $L_{i}$. The statistical model for data analysis becomes:

$$
Y_{i, t}=\alpha+\beta_{0} X_{i, t}+\beta_{1} X_{i, t-1}+\beta_{2} X_{i, t-2}+\ldots+\beta_{n} X_{i, t-n}+L_{i}+e_{i, t}
$$

where the $i, t$ subscript refers to the response, predictor, or residual for the $i$ th location $(i=1, \ldots, 6)$ at the $t$ th time. The location effect accounts for such things as differences in overall background inoculum densities among the locations or possible (unrecorded) differences in experimental methodology. All terms in the model are fixed effects, except for the residual $(e)$ which is a random effect. It is assumed that $e$ is normally distributed with mean 0 and fixed variance $\sigma^{2}$. Although spore counts are discrete, when the numbers are generally large (as in our data), the counts on a log scale are well approximated by a continuous distribution (52).

A PDL regression analysis (7,8,55,62; Appendix), based on equation 2, was performed using PROC PDLREG of SAS (SAS Institute, Cary, NC [33]) to determine the structure and form of the relationship between $Y$ and the weather variables. This involved (i) determining the time window (time lag length, $n$ ) over which a weather variable exhibited a significant effect on $Y$, and (ii) determining the manner, if any, in which the parameters $(\beta s)$ systematically changed with time lag (i.e., the order of the polynomial). The first phase of the PDL analysis has some similarity to the methodology of Coakley et al. (11) and Francl et al. (22) for relating weather in defined time windows to the biological response variable of interest. However, the PDL approach allows for day-specific weighting of variables within windows and serial correlations of the predictor variables. In the first phase of the PDL analysis, the lag length was determined for each of 49 weather variables. This entailed fitting equation 2 to each weather variable separately for lag lengths of 0 to 8 days. The $L_{i}$ term in equation 2 was fitted through the use of "dummy" (indicator) variables for locations in the PDLREG procedure. A maximum lag length of 8 days was chosen because of the available weather data and the lengths of the time series for the location-years. Models with unconstrained parameters were fitted in this phase of the analysis because fixing the polynomial degree ( $d$; Appendix) with varying lag $(n)$ biases the derived goodness-of-fit statistics (42). Model appraisal was based on mean square error (MSE) and Akaike's information criterion (AIC) (52).

In the second phase of PDL model fitting, the polynomial degree $(d)$ was determined for each of the selected weather variables using the fixed lag length that was selected in the first phase of analysis. This was done utilizing the method incorporated into the PDLREG procedure based on orthogonal polynomials (Appendix). A polynomial term was considered significant (and the term retained) if the absolute value of its associated student $t$ statistic was greater than 1.5.

Mixed model analysis. Results from the PDL modeling indicated that there was serial correlation of the residuals (L. V. Madden, unpublished data). Although direct PDL analysis easily allows certain approaches for correcting this serial correlation, a more general approach was taken here, which can accommodate explicitly serial correlation within location-years, between location-year variability, and allow for more convenient inclusion of multiple weather variables in the same model. Based on the PDL results for each weather variable and the location categorical variable, composite weather variables were constructed $\left(X^{*}\right)$ as explained above for equation 1c. That is, the weights $\left(w_{m}\right)$ were calculated from the $\beta_{m}$ values, based on the selected $n$ and $d$ for the model, and the composite variable was defined as

$$
X_{i, t}^{*}=w_{0} X_{i, t}+w_{1} X_{i, t-1}+w_{2} X_{i, t-2} \cdots+w_{n} X_{i, t-n}
$$

A linear mixed model $(24,25,33)$ was fitted to the data for the weather variables that explained the most variability in $Y$ in the PDL modeling (i.e., for those variables that resulted in models with relatively low MSE and AIC). One or more predictor weather variables were included. If we let $X_{(1)}^{*}$, and $X_{(2)}^{*}$, represent two of the composite weather variables, for instance, then the linear mixed model is given as

$$
Y_{i, j, t}=\alpha+\beta_{(1)}^{*} X_{(1) i, j, t}^{*}+\beta_{(2)}^{*} X_{(2) i, j, t}^{*}+L_{i}+T(L)_{i, j}+e_{i, j, t}
$$

where the $i, j, t$ subscript represents the $i$ th location, $j$ th year within the $i$ th location, and $t$ is time within the location-year. $L_{i}$ is the location effect, as before, and $T(L)_{i, j}$ is the random effect of year within the location, and $e_{i j t}$ is, as before, the residual (a random effect). Except for $T(L)$ and $e$, all terms in the model are fixed effects. It is assumed that $T(L)$ is normally distributed with mean 0 and variance $\sigma_{T(L)}^{2}$, and that $e$ is normally distributed with mean 0 and variance-covariance matrix $\boldsymbol{R}$. Through the mixed model, several possible serial (auto-) correlation structures for the residuals can be specified to account for the temporal dependency of the data (52). It is further assumed that $T(L)$ and $e$ are independent, a standard assumption of mixed model analysis.

Equation 4 (with any chosen number of predictor variables $\left.\left[X^{*}\right]\right)$ was fitted to the data using PROC MIXED of SAS using the restricted maximum likelihood method. For a given set of $X^{*}$ variables, the following structures for $\boldsymbol{R}$ were evaluated: independence (no serial correlation of $e$ ); compound symmetry (fixed correlation of $e$ between all pairs of times within a location-year); first-order autoregressive (geometrically declining correlation of $e$ between pairs of times as the temporal separation of the pairs increases within a location-year); and banded Toeplitz (unique correlation of $e$ between pairs of times for each time-pair separation within a location-year). The banded Toeplitz structure can be viewed as a moving-average structure of the residuals. We examined moving-averages up to an order of 9 for the Toeplitz structure. The AIC criterion was used to select the most appropriate structure for $\boldsymbol{R}$ (including the order of the banded Toeplitz structure).

After selection of the most appropriate residual covariance structure, the fixed effects were tested with $F$ tests. With the estimates of the fixed effects (coefficients for the composite weather variables [e.g., $\left.\hat{\beta}_{(1)}^{*}\right]$ and for locations), five models consisting of different combinations of composite weather variables were developed and used to predict log spore density. Predictions of $Y$ (and confidence intervals for the predictions) were determined utilizing the estimated fixed-effect and random-effect terms. Accuracy of predictions was determined using the so-called concordance correlation coefficient (CCC) $(32,39)$. CCC simultaneously provides a measure of precision (variability) and bias of the predictions (34), quantified as the product of: the Pearson product-moment correlation between $Y$ and $\hat{Y}$ ( $r$; for precision); and the $C_{b}$ bias coefficient (a measure of the proximity of best fitting regression line between $Y$ and $\hat{Y}$ to the line of perfect fit).

Mixed model evaluation. Appropriateness of the fitted mixed models was assessed using the residuals and various influence statistics $(30,33)$. Studentized residuals were plotted versus the predicted values to determine if there was a random pattern; quantile-quantile plots of the residuals were also made to determine if normality was a reasonable assumption.

Influence statistics are used to determine the degree to which the fit of a model, the model prediction, or the estimates of the 
parameters are affected by individual data points or groups of data points (33). Here, we specifically determined the influence of each location-year on the modeling results. This allowed us to determine if the identified overall relationship between $Y$ and weather variables (as quantified through the models) was unduly influenced by certain location-year combinations. Among other things, these statistics can be used to determine if the models developed required adjustment for particular situations not included in the model. Options in the MIXED procedure of SAS (for version 9.1 and later) permit calculation of all these statistics directly.

For this study, the following influence statistics were determined for each location-year: likelihood distance function $(l$; an overall measure of the effect of each group on model fit); Cook's
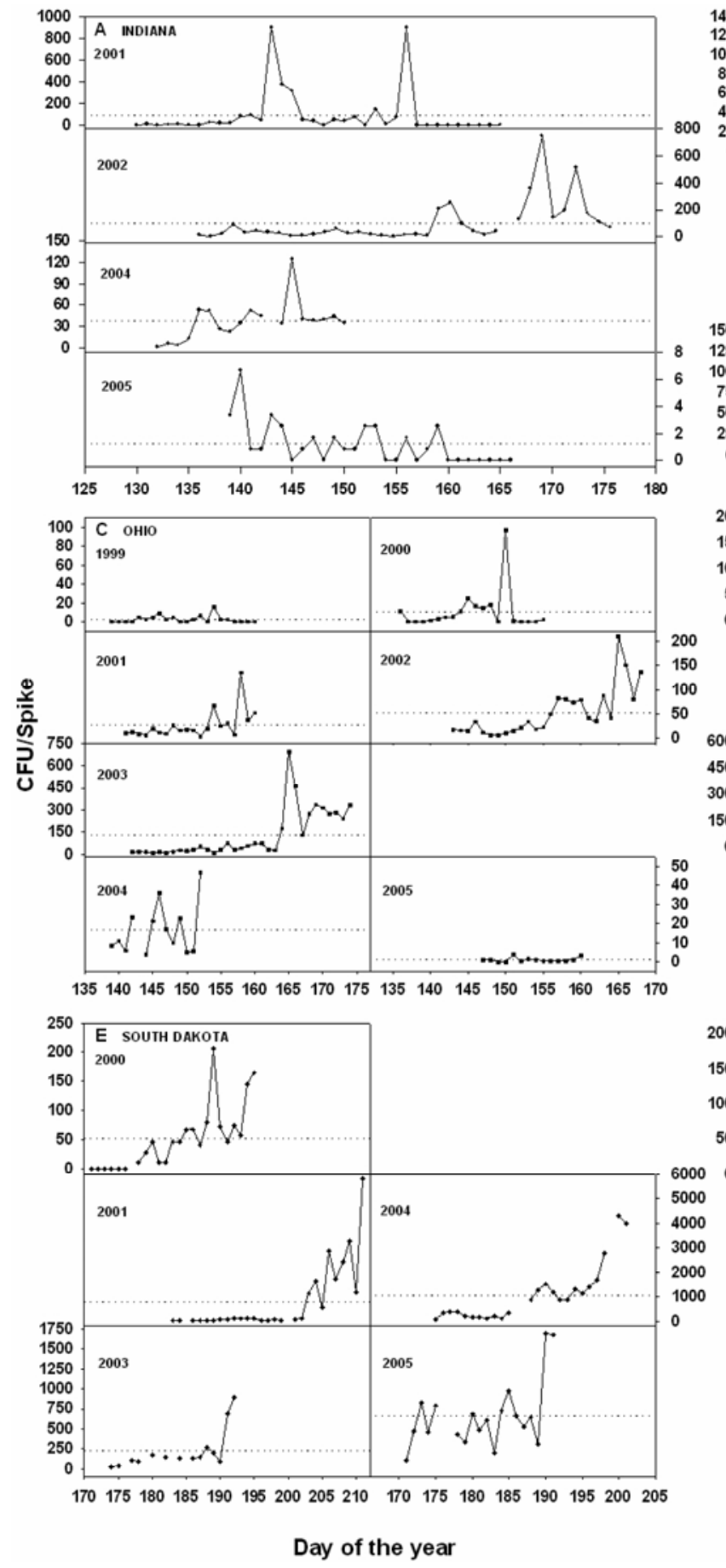

distance $\left(D_{P}\right.$; a normalized measure of the effect of each group on the vector (set) of fixed-effect parameter estimates; and Cook's distance for the random-effect terms $\left(D_{v}\right.$; a normalized measure of the effect of each group on the quantified variability and covariance).

\section{RESULTS}

Spike sampling. The mean daily number of propagules of $G$. zeae recovered from wheat spikes, quantified as CFUs per spike, varied from one location to another and from year to year within a location (Table 1, Fig. 2). For any given location-year, there was substantial day-to-day variation in the number of CFUs per spike (Fig. 2). Based on averages across all days and years that samples
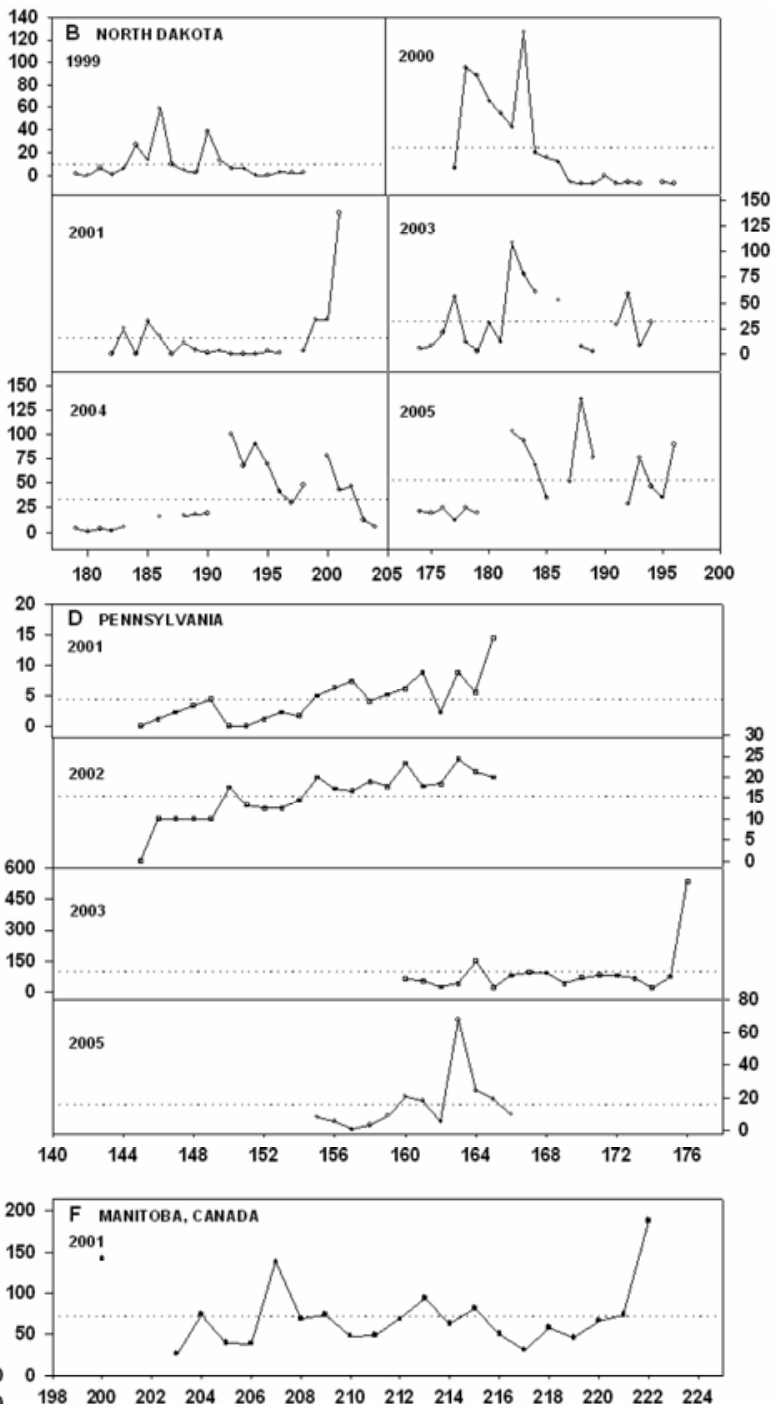

Day of the year South Dakota, and F, Manitoba, Canada. 
were collected in a location, the highest overall mean daily number of propagules per location was observed for South Dakota (592 CFU/spike), followed by Manitoba ( $72 \mathrm{CFU/spike),} \mathrm{Indiana}$ (65 CFU/spike), Ohio (45 CFU/spike), Pennsylvania (31 CFU/ spike), and finally North Dakota (19 CFU/spike). The large difference in means among locations is a justification for the location effect $\left(L_{i}\right)$ in the PDL and mixed models (equations 2 and 4). For each location, the lowest and highest mean daily counts occurred in 2005 and 2002 in Indiana (Fig. 2A); 1999 and 2005 in North Dakota (Fig. 2B); 2005 and 2003 in Ohio (Fig. 2C); 2001 and 2003 in Pennsylvania (Fig. 2D); and 2000 and 2004 in South Dakota (Fig. 2E).

There was no consistent trend of $Y$ versus time within the location-years. In some cases, $Y$ generally increased over time (e.g., Pennsylvania in 2001 and 2002), whereas in other cases, $Y$ generally decreased over time (e.g., Indiana in 2005). In many other cases, there was no apparent trend in $Y$ versus time.

PDL analysis: Determining the lag length. The results from the fit of equation 2 to each of the 49 weather variables for lags of 0 to 8 days are summarized in Table 2. Both MSE and AIC were used as guides for identifying the most influential weather variables and determining the time lag over which each variable had significant influence on $Y$ (log-transformed CFU/spike). The lag length that resulted in the lowest MSE and AIC was considered to provide the best fit, and was selected as the appropriate lag length for further analysis. This time lag length represents the time window in days that best described the temporal influence of the weather variable in question on $Y$. In general, for all 49 weather variables, models fitted without lags $(n=0)$ had the highest MSE and AIC relative to models fitted with lags. Increasing the lag length from 0 to 8 (that is, accounting for the influence of weather conditions up to 8 days prior to the day spikes were sampled) resulted in a decrease in MSE and especially AIC. For instance, for relative humidity-related variables, models fitted for a lag of 0 days had AIC values ranging from 2,218 to 2,250, but models for a lag of 8 days had AIC values from 2,075 to 2,144 . For most weather variables, there was a monotonic decrease in the fit statistics over 8 lag times. However, for a few variables (e.g., NCT930) there was a slight increase in these statistics around lags of 4 days, followed by a general decrease through 8 days.

In general, for 8-day lags (plus the current day), temperature and relative humidity variables representing conditions of the air above and around the canopy provided a better fit than variables representing conditions within the canopy (Table 2). For instance, average relative humidity of the air (AVARH) yielded lower MSE and AIC than average relative humidity within the canopy $(\mathrm{AVCRH})$. For both air temperature and relative humidity, variables summarized as daily averages provided a slightly better fit than variables summarized to represent the number of hours of temperature or relative humidity within specific ranges for each day. For instance, considering just 8-day lags, the model with average daytime air temperature (AVDAT), with an MSE of 2.68 and an AIC of 2,176, provided a better fit than the model with number of hours per day with the air temperature between 15 and $30^{\circ} \mathrm{C}$ (AT1530), with an MSE of 2.71 and an AIC of 2,182. Similarly, the use of AVARH yielded a model with lower MSE and AIC than the model with the number of hours per day with air RH $>90 \%$ (ARH90).

Relative to temperature- and wind-related variables, moisturerelated variables (relative humidity, rainfall, and wetness durations) provided better fits to the data. For a lag of 8 days, AVARH, rainfall intensity, and daily wetness duration (DWD) models generally had lower MSE and AIC values than temperature and wind models (Table 2).

PDL analysis: Determining the polynomial degree. Based on MSE and AIC, a time window of 8 days was chosen as the appropriate lag length $(n=8$ in equation 2$)$ for describing the relationship between all weather conditions and the log of spore density on the wheat spike $(Y)$. The appropriate polynomial degree $(d)$ of equation A1 in the Appendix for representing the $\beta_{m}$ parameters of equation 2 was determined with Student $t$ tests of the derived orthogonal polynomial terms (which are, in turn, functions of the $\eta_{k}$ parameters of equations A2 and 3). The significant polynomial degree was never above 2 (quadratic) for any of the weather variables, based on magnitude of the $t$ statistics. That is, all polynomial terms from 3 to 8 had associated $t$ statistics less than 1.5 in absolute value. Table 3 shows the $t$ statistics for polynomial degrees, $d$, of 0,1 , and 2 for a subset of the weather variables, which generally provided the best fits to the data based on MSE and AIC (Table 2). The highest order polynomial term with a $t$ statistic above 1.5 in absolute value was considered the appropriate polynomial degree.

For all of the selected temperature-related variables, with the exception of the variable representing the number of hours of daytime air temperature between 15 and $30^{\circ} \mathrm{C}$ (DAT1530), the second-degree power was the highest order term that was significantly different from zero, suggesting that the quadratic polynomial was most appropriate. This means that the $\beta_{m}$ lag coefficients of equation 2 changed with lag $m(m=0, \ldots, 8)$ in a quadratic fashion; the $\beta_{m}$ values could either increase to a maximum value between 0 and 8 , and then decline again, or they could decrease to a minimum value between 0 and 8 and then increase again. Conversely, for all relative humidity-related variables and daily wetness duration (DWD), the second-order power was not significantly different from zero, whereas the first-order power was significant for all but the variable representing the number of hours of nighttime air relative humidity $>90 \%$ (NARH90). This suggested that the first-order polynomial was appropriate for relative humidity and wetness variables. This means that the $\beta_{m}$ values monotonically decreased from 0 to 8 lags or monotonically increased from 0 to 8 lags. For variables representing rainfall and a combination of hours of air temperature greater than $15^{\circ} \mathrm{C}$ and relative humidity $>90 \%$ (AT15RH90), neither the first nor second order powers were significant, indicating that a polynomial of power 0 was appropriate. This means that the $\beta_{m}$ values did not change with lag (i.e., $\beta_{0}=\beta_{1}=\ldots=\beta_{8}$ ). A significant high-order polynomial degree term (e.g., at $d=6$ ), not found here for any variable, would have indicated that the $\beta_{m}$ values did not change smoothly with lag (which could happen if weather conditions on only specific days had a large influence on $Y$ ).

Figure 3 shows the lag distributed coefficients $\left(\beta_{m}\right)$ for a subset of the weather variables plotted against the corresponding lag lengths. The association between temperature (expressed as AT1530, AVDAT, and AVAT, Fig. 3A, B, and C) and $Y$ varied over time, with the estimated $\beta_{m}$ parameters for AT1530, AVDAT, and AVAT starting at negative values at lags of 0 and 1 days, increasing to a maximum value at a lag of 5 days, and decreasing towards 0 at higher lags. Parameter estimates at lags of 0,1 , and 3 to 7 days were significant. These results suggest that an increase in average air temperature on the day of sampling and the first day prior to sampling was associated with a decrease in $Y$, whereas an increase in temperature between 3 and 8 days prior to sampling was associated with an increase in $Y$. The overall (i.e., marginal) effect of the temperature variables $\left(\beta^{*}=\Sigma \beta_{m}\right)$ on $Y$ was positive (Fig. 3A, B, and C), however, meaning that an increase in AT1530, AVDAT, or AVAT resulted in an increase in $Y$ (although this increase was spread out over 9 days). In other words, the short-term reduction in $Y$ at days 0 and 1 would be more than offset by the increase in $Y$ on subsequent days.

Relative humidity, represented as AVARH or AVDARH in Figure 3, had a significant, positive association with $Y$ for all time lags (Fig. 3D and E). However, the magnitude of the effect (as quantified by $\beta_{m}$ ) decreased linearly over time, with the greatest effect occurring at $m=0$ and the lowest at $m=8$. Similarly, the magnitude of the association between DWD and $Y$ also decreased 
linearly over time, with a significant, positive effect at lags from 0 to 4 days, no significant effect at a lag of 5 days, and a significant, negative effect at lags from 6 to 8 days (Fig. 3F). Rainfall intensity and AT15RH90, on the other hand, had significant, positive effects on $Y$ that were constant over all 9 days (Fig. 3G and $\mathrm{H}$ ). The marginal effects ( $\beta^{*}$ values) were positive for all relative humidity, wetness duration, and rainfall variables.
Mixed model analysis. Mixed models of the form of equation 4 , containing two or three of the composite predictor variables identified using the PDL regression results (equation 3), were fitted to the data in the second stage of the analysis. The models shown accounted for the joint effects of relative humidity, temperature, and rainfall on $Y$. The wetness-duration variable (DWD) was not used because wetness was only measured in about half of

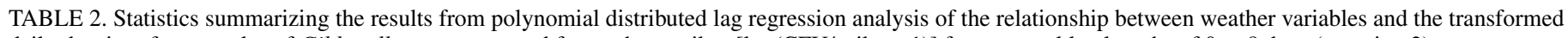
daily density of propagules of Gibberella zeae recovered from wheat spikes $[\log (\mathrm{CFU} / \mathrm{spike}+1)]$ for temporal lag lengths of 0 to 8 days $($ equation 2$)$

\begin{tabular}{|c|c|c|c|c|c|c|c|c|c|}
\hline \multirow[b]{2}{*}{ Variables $^{\mathrm{a}}$} & \multicolumn{9}{|c|}{ Lag length (days) ${ }^{\mathrm{b}}$} \\
\hline & 0 & 1 & 2 & 3 & 4 & 5 & 6 & 7 & 8 \\
\hline СТ9 & $2.83 / 2259^{c}$ & $2.82 / 2252$ & $2.80 / 2245$ & $2.80 / 2246$ & $2.81 / 2240$ & $2.82 / 2236$ & $2.83 / 2232$ & $2.79 / 2208$ & $2.75 / 2191$ \\
\hline СТ930 & $2.82 / 2257$ & $2.81 / 2250$ & $2.79 / 2241$ & $2.79 / 2243$ & $2.80 / 2239$ & $2.81 / 2235$ & $2.82 / 2230$ & $2.78 / 2206$ & $2.74 / 2188$ \\
\hline CT1530 & $2.83 / 2260$ & $2.82 / 2251$ & $2.80 / 2244$ & $2.79 / 2243$ & $2.79 / 2236$ & $2.79 / 2229$ & $2.79 / 2223$ & $2.74 / 2198$ & $2.71 / 2182$ \\
\hline AVCT & $2.80 / 2254$ & $2.80 / 2248$ & $2.79 / 2243$ & $2.78 / 2241$ & $2.76 / 2231$ & $2.77 / 2225$ & $2.77 / 2219$ & $2.72 / 2194$ & $2.70 / 2179$ \\
\hline DCT9 & $2.83 / 2256$ & $2.83 / 2254$ & $2.81 / 2247$ & $2.82 / 2249$ & $2.82 / 2243$ & $2.84 / 2239$ & $2.84 / 2234$ & $2.80 / 2210$ & $2.77 / 2193$ \\
\hline DCT930 & $2.79 / 2250$ & $2.79 / 2246$ & $2.78 / 2240$ & $2.78 / 2241$ & $2.79 / 2236$ & $2.79 / 2231$ & $2.80 / 2226$ & $2.75 / 2200$ & $2.71 / 2181$ \\
\hline DCT1530 & $2.83 / 2259$ & $2.83 / 2253$ & $2.82 / 2248$ & $2.81 / 2248$ & $2.83 / 2244$ & $2.83 / 2237$ & $2.83 / 2232$ & $2.79 / 2208$ & $2.76 / 2191$ \\
\hline AVDCT & $2.77 / 2248$ & $2.78 / 2243$ & $2.77 / 2239$ & $2.76 / 2238$ & $2.75 / 2228$ & $2.75 / 2222$ & $2.75 / 2214$ & $2.70 / 2190$ & $2.68 / 2174$ \\
\hline NCT9 & $2.83 / 2258$ & $2.82 / 2252$ & $2.80 / 2244$ & $2.80 / 2246$ & $2.81 / 2240$ & $2.82 / 2235$ & $2.83 / 2231$ & $2.78 / 2207$ & $2.75 / 2190$ \\
\hline NCT930 & $2.83 / 2258$ & $2.82 / 2252$ & $2.80 / 2243$ & $2.80 / 2245$ & $2.81 / 2240$ & $2.82 / 2236$ & $2.83 / 2233$ & $2.79 / 2208$ & $2.76 / 2191$ \\
\hline NCT1530 & $2.82 / 2259$ & $2.82 / 2251$ & $2.80 / 2244$ & $2.78 / 2241$ & $2.76 / 2231$ & $2.77 / 2225$ & $2.76 / 2217$ & $2.71 / 2192$ & $2.68 / 2176$ \\
\hline AVNCT & $2.82 / 2258$ & $2.82 / 2251$ & $2.80 / 2245$ & $2.79 / 2244$ & $2.77 / 2233$ & $2.78 / 2227$ & $2.78 / 2222$ & $2.73 / 2197$ & $2.71 / 2182$ \\
\hline AT9 & $2.83 / 2258$ & $2.83 / 2253$ & $2.81 / 2247$ & $2.81 / 2248$ & $2.83 / 2243$ & $2.84 / 2239$ & $2.84 / 2234$ & $2.80 / 2210$ & $2.77 / 2193$ \\
\hline AT930 & $2.82 / 2258$ & $2.83 / 2253$ & $2.81 / 2245$ & $2.81 / 2247$ & $2.82 / 2243$ & $2.83 / 2238$ & $2.84 / 2234$ & $2.80 / 2210$ & $2.76 / 2192$ \\
\hline AT1530 & $2.82 / 2259$ & $2.82 / 2252$ & $2.80 / 2243$ & $2.78 / 2242$ & $2.78 / 2234$ & $2.78 / 2227$ & $2.78 / 2221$ & $2.73 / 2197$ & $2.71 / 2182$ \\
\hline AVAT & $2.81 / 2256$ & $2.81 / 2249$ & $2.80 / 2243$ & $2.78 / 2241$ & $2.76 / 2230$ & $2.76 / 2224$ & $2.76 / 2218$ & $2.71 / 2193$ & $2.69 / 2178$ \\
\hline DAT9 & $2.83 / 2256$ & $2.83 / 2253$ & $2.82 / 2245$ & $2.82 / 2250$ & $2.83 / 2245$ & $2.85 / 2241$ & $2.85 / 2236$ & $2.81 / 2213$ & $2.78 / 2196$ \\
\hline DAT930 & $2.82 / 2255$ & $2.82 / 2252$ & $2.81 / 2246$ & $2.82 / 2248$ & $2.82 / 2243$ & $2.83 / 2238$ & $2.84 / 2234$ & $2.79 / 2208$ & $2.75 / 2190$ \\
\hline DAT1530 & $2.82 / 2258$ & $2.83 / 2253$ & $2.81 / 2246$ & $2.80 / 2245$ & $2.81 / 2240$ & $2.80 / 2233$ & $2.80 / 2226$ & $2.76 / 2203$ & $2.74 / 2188$ \\
\hline AVDAT & $2.80 / 2255$ & $2.80 / 2248$ & $2.79 / 2243$ & $2.78 / 2241$ & $2.76 / 2230$ & $2.76 / 2223$ & $2.75 / 2215$ & $2.71 / 2191$ & $2.68 / 2176$ \\
\hline NAT9 & $2.83 / 2258$ & $2.83 / 2253$ & $2.81 / 2246$ & $2.81 / 2248$ & $2.82 / 2243$ & $2.83 / 2238$ & $2.84 / 2233$ & $2.79 / 2209$ & $2.76 / 2193$ \\
\hline NAT930 & $2.83 / 2258$ & $2.83 / 2253$ & $2.80 / 2245$ & $2.81 / 2247$ & $2.82 / 2242$ & $2.83 / 2238$ & $2.84 / 2233$ & $2.80 / 2210$ & $2.76 / 2193$ \\
\hline NAT1530 & $2.82 / 2258$ & $2.82 / 2251$ & $2.80 / 2243$ & $2.78 / 2242$ & $2.76 / 2231$ & $2.77 / 2225$ & $2.77 / 2219$ & $2.72 / 2195$ & $2.70 / 2179$ \\
\hline AVNAT & $2.82 / 2257$ & $2.82 / 2251$ & $2.80 / 2245$ & $2.79 / 2243$ & $2.77 / 2232$ & $2.77 / 2226$ & $2.78 / 2220$ & $2.72 / 2195$ & $2.70 / 2180$ \\
\hline MAXAT & $2.80 / 2254$ & $2.80 / 2247$ & $2.80 / 2244$ & $2.79 / 2243$ & $2.77 / 2233$ & $2.78 / 2227$ & $2.78 / 2220$ & $2.73 / 2196$ & $2.70 / 2180$ \\
\hline CRH90 & $2.74 / 2242$ & $2.70 / 2226$ & $2.67 / 2217$ & $2.66 / 2216$ & $2.66 / 2208$ & $2.64 / 2199$ & $2.64 / 2191$ & $2.58 / 2165$ & $2.54 / 2144$ \\
\hline AVCRH & $2.66 / 2226$ & $2.60 / 2204$ & $2.56 / 2193$ & $2.53 / 2186$ & $2.52 / 2177$ & $2.49 / 2165$ & $2.49 / 2158$ & $2.43 / 2129$ & $2.38 / 2108$ \\
\hline DCRH90 & $2.74 / 2239$ & $2.68 / 2223$ & $2.63 / 2209$ & $2.62 / 2206$ & $2.61 / 2198$ & $2.61 / 2190$ & $2.61 / 2184$ & $2.56 / 2160$ & $2.52 / 2140$ \\
\hline AVDCRH & $2.66 / 2226$ & $2.60 / 2204$ & $2.55 / 2189$ & $2.52 / 2183$ & $2.51 / 2175$ & $2.49 / 2164$ & $2.48 / 2157$ & $2.42 / 2127$ & $2.37 / 2105$ \\
\hline NCRH90 & $2.77 / 2246$ & $2.74 / 2235$ & $2.73 / 2229$ & $2.72 / 2228$ & $2.72 / 2221$ & $2.70 / 2211$ & $2.69 / 2203$ & $2.63 / 2174$ & $2.58 / 2153$ \\
\hline AVNCRH & $2.70 / 2235$ & $2.65 / 2216$ & $2.63 / 2207$ & $2.60 / 2202$ & $2.59 / 2193$ & $2.57 / 2182$ & $2.56 / 2174$ & $2.50 / 2147$ & $2.47 / 2128$ \\
\hline ARH90 & $2.70 / 2234$ & $2.63 / 2211$ & $2.57 / 2195$ & $2.54 / 2189$ & $2.51 / 2176$ & $2.48 / 2163$ & $2.46 / 2152$ & $2.40 / 2123$ & $2.34 / 2098$ \\
\hline AVARH & $2.62 / 2218$ & $2.54 / 2190$ & $2.48 / 2174$ & $2.43 / 2163$ & $2.41 / 2152$ & $2.37 / 2137$ & $2.36 / 2127$ & $2.29 / 2097$ & $2.24 / 2075$ \\
\hline DARH90 & $2.71 / 2233$ & $2.65 / 2216$ & $2.58 / 2197$ & $2.56 / 2193$ & $2.53 / 2181$ & $2.52 / 2172$ & $2.51 / 2162$ & $2.45 / 2135$ & $2.40 / 2112$ \\
\hline DARH80 & $2.67 / 2228$ & $2.60 / 2204$ & $2.54 / 2188$ & $2.50 / 2179$ & $2.48 / 2168$ & $2.46 / 2156$ & $2.44 / 2147$ & $2.38 / 2117$ & $2.33 / 2096$ \\
\hline AVDARH & $2.63 / 2220$ & $2.55 / 2194$ & $2.49 / 2176$ & $2.44 / 2166$ & $2.43 / 2156$ & $2.40 / 2142$ & $2.38 / 2133$ & $2.31 / 2102$ & $2.26 / 2078$ \\
\hline NARH90 & $2.73 / 2238$ & $2.66 / 2218$ & $2.62 / 2206$ & $2.59 / 2201$ & $2.57 / 2189$ & $2.54 / 2175$ & $2.52 / 2165$ & $2.45 / 2135$ & $2.40 / 2112$ \\
\hline AVNARH & $2.65 / 2224$ & $2.56 / 2196$ & $2.51 / 2182$ & $2.47 / 2173$ & $2.45 / 2162$ & $2.42 / 2148$ & $2.40 / 2138$ & $2.34 / 2110$ & $2.30 / 2089$ \\
\hline MAXARH & $2.75 / 2244$ & $2.70 / 2226$ & $2.66 / 2214$ & $2.63 / 2209$ & $2.63 / 2202$ & $2.63 / 2196$ & $2.63 / 2190$ & $2.60 / 2168$ & $2.57 / 2151$ \\
\hline MINARH & $2.66 / 2226$ & $2.57 / 2198$ & $2.53 / 2185$ & $2.47 / 2173$ & $2.44 / 2160$ & $2.42 / 2148$ & $2.41 / 2139$ & $2.34 / 2108$ & $2.29 / 2087$ \\
\hline CT15RH90 & $2.80 / 2255$ & $2.76 / 2239$ & $2.70 / 2224$ & $2.66 / 2216$ & $2.63 / 2203$ & $2.59 / 2186$ & $2.56 / 2173$ & $2.48 / 2141$ & $2.42 / 2118$ \\
\hline AT15RH90 & $2.78 / 2250$ & $2.72 / 2231$ & $2.66 / 2215$ & $2.61 / 2205$ & $2.57 / 2189$ & $2.53 / 2174$ & $2.50 / 2160$ & $2.42 / 2127$ & $2.36 / 2103$ \\
\hline DWD & $2.69 / 1582$ & $2.64 / 1558$ & $2.63 / 1542$ & $2.61 / 1528$ & $2.56 / 1507$ & $2.53 / 1489$ & $2.49 / 1465$ & $2.40 / 1424$ & $2.34 / 1398$ \\
\hline TOTAL & $2.80 / 2256$ & $2.78 / 2242$ & $2.75 / 2234$ & $2.73 / 2230$ & $2.73 / 2224$ & $2.73 / 2218$ & $2.71 / 2207$ & $2.64 / 2177$ & $2.58 / 2153$ \\
\hline DURATION & $2.81 / 2254$ & $2.79 / 2246$ & $2.78 / 2239$ & $2.77 / 2239$ & $2.77 / 2233$ & $2.78 / 2227$ & $2.77 / 2218$ & $2.70 / 2190$ & $2.64 / 2167$ \\
\hline INTENSITY & $2.80 / 2252$ & $2.75 / 2238$ & $2.71 / 2226$ & $2.67 / 2219$ & $2.67 / 2212$ & $2.66 / 2203$ & $2.63 / 2190$ & $2.57 / 2162$ & $2.51 / 2138$ \\
\hline AVDWS & $2.68 / 1887$ & $2.68 / 1886$ & $2.69 / 1884$ & $2.69 / 1886$ & $2.70 / 1884$ & $2.69 / 1880$ & $2.70 / 1879$ & $2.65 / 1858$ & $2.61 / 1846$ \\
\hline DMAXWS & $2.67 / 1887$ & $2.68 / 1884$ & $2.68 / 1882$ & $2.68 / 1884$ & $2.69 / 1882$ & $2.69 / 1880$ & $2.70 / 1878$ & $2.65 / 1859$ & $2.61 / 1845$ \\
\hline MINWS & $2.82 / 2258$ & $2.82 / 2252$ & $2.82 / 2249$ & $2.82 / 2250$ & $2.83 / 2245$ & $2.84 / 2239$ & $2.84 / 2233$ & $2.78 / 2207$ & $2.75 / 2189$ \\
\hline
\end{tabular}

${ }^{a}$ Codes representing summarized weather variables. In the codes for temperature (T) and relative humidity (RH) variables (the first 42 variables in the table), $\mathrm{C}$, A, $\mathrm{D}, \mathrm{N}, \mathrm{AV}, \mathrm{MAX}$, and MIN represent canopy, air, daytime, nighttime, average, maximum, and minimum, respectively. The numbers $9,15,930$, and 1530 in the codes for temperature variable represent temperatures $>9^{\circ} \mathrm{C},>15$, between 9 and $30^{\circ} \mathrm{C}$, and between 15 and $30^{\circ} \mathrm{C}$, respectively, whereas the numbers 80 and 90 in codes for $\mathrm{RH}$ variables represent $\mathrm{RH}>80$ and $>90 \%$, respectively. Variables for daytime periods represent summaries of conditions between 600 and $1800 \mathrm{~h}$, whereas variables for nighttime periods represent summaries of conditions between 1800 and $600 \mathrm{~h}$. With the exception of averages, MAX and MIN, which are given in ${ }^{\circ} \mathrm{C}$ or \%, all temperature and RH variables are given in terms of number of hours. So, for example, the codes DCT1530 and AVNARH indicate daytime canopy temperature between 15 and $30^{\circ} \mathrm{C}$ and average nighttime air relative humidity, respectively. DWD $=$ daily wetness duration (h), TOTAL $=$ total daily rainfall $(\mathrm{mm})$, DURATION $=$ daily rainfall duration $(\mathrm{h})$, INTENSITY $=$ daily rainfall intensity $(\mathrm{mm} / \mathrm{h})$, AVDWS $=$ average daily wind speed $(\mathrm{m} / \mathrm{s})$, DMAXWS $=$ daily maximum wind speed $(\mathrm{m} / \mathrm{s})$, and MINWS $=$ minimum daily wind speed $(\mathrm{m} / \mathrm{s})$.

${ }^{\mathrm{b}} \mathrm{Lag}$ length $=$ time windows of $n=0$ to 8 days before the day spores were samples. Separate polynomial distributed lag regression models (with no constraint on the polynomial degree; i.e., $d=n$ ) were fitted to each weather variable for each lag length using 581 observations collected from Indiana (118), Manitoba (21), North Dakota (115), South Dakota (108), Ohio (148), and Pennsylvania (71).

${ }^{c}$ For each lag length by weather combination, the statistics represent mean square error and Akaike's information criteria. Both statistics adjust for number of parameters in the model, and in both cases, the smaller the statistic, the better the fit. 
the location-years. Although several relative humidity-based variables provided the best fits to the data using PDL, these variables were, by definition, highly intercorrelated (since they were functionally related, being constructed with the same daily or hourly weather measurements). Thus, using more than one composite variable based on moisture would not necessarily add to the goodness of fit of the model and would result in lower precision of the estimated parameters (30). Likewise, using more than one temperature or rainfall variable in a model would have similar problems. There can be correlations between temperature, moisture, and rainfall, of course, but these are expected to be less. Results for five mixed models are presented.

Through mixed model analysis, the between-location-year variability and the serial correlation of observations within locationyears was evaluated by fitting the model with and without the year within location random effect $(T(L))$, and with different error (residual) correlation structures, and comparing the AIC values (which were, in turn, based on -2 times the log-likelihoods). Results are given here for model M1 (described below). With no $T(L)$ term and a single residual variance term (the "simple" error structure, which means an assumption of 0 serial correlation), the AIC was 2052.7. With inclusion of $T(L)$ and a single residual variance (compound symmetry error structure, i.e., fixed serial correlation of all residuals within location-years), the AIC was 1810.5. The $T(L)$ random effect was highly significant $(P<0.01)$ and included in all other more complicated models described later. With a first-order autoregressive residual structure (geometrically declining serial correlation as the differences in time pairs increased), the AIC was 1634.9, and with a so-called banded
Toeplitz structure or order 9 (representing a moving-average structure of the residuals of order 8), the AIC was 1606.6. However, the highest two terms of this moving average were not significant, so a banded Toeplitz of order 7 was fitted; this model resulted in an AIC of 1604.7, the lowest value found. Thus, the banded Toeplitz of order 7 was selected as the error correlation structure for all further analyses. With this structure, there was a positive serial correlation of the residuals, conditional on the location-year, for time lags up to 6 days. Very similar results were found for all five models considered.

Mixed model M1 contained the 9-day composites of AVARH (AVARH ${ }^{*}$, equation 3), AVDAT (AVDAT ${ }^{*}$ ), and INTENSITY (INTENSITY*) as fixed-effects terms, together with the location fixed effect and year-within-location random effect (equation 4 for generic version of this model). These three weather variables provided the overall best fits in the individual PDL regression analysis in the categories of moisture, temperature, and rainfall (Table 3). All estimated parameters (the $\beta^{*}$ marginal effect parameters or coefficients) were highly significant $(P<0.01)$ and positive. That is, increasing values of AVARH*, AVDAT*, and INTENSITY* ${ }^{*}$ resulted in increasing $Y$, although the effects were spread out over 9 days (lags of 0 to 8 ).

Models M2 through M5 substituted either AVDARH* for AVARH $^{*}, \mathrm{AT}^{2} 530^{*}$ for AVDAT*, or the temperature-moisture variable AT15RH90* for the moisture and temperature variables. The values of $\beta^{*}$ for all models were somewhat different from those found in the PDL regressions. There are two major reasons for this. First, the mixed models accounted for the simultaneous estimation of all fixed-effect parameters considered, whereas the

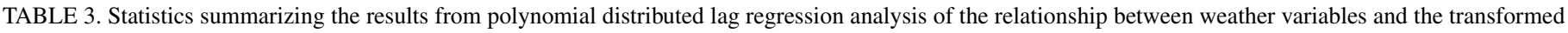

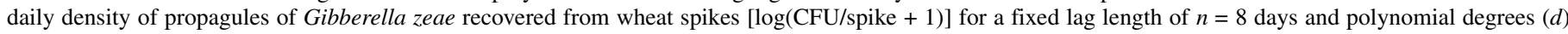
of 0,1 , and 2 (Appendix)

\begin{tabular}{|c|c|c|c|c|}
\hline \multirow[b]{2}{*}{ Variables $^{\mathrm{a}}$} & \multicolumn{3}{|c|}{ Polynomial degree $^{b}$} & \multirow[b]{2}{*}{$\mathrm{CCC}^{\mathrm{c}}$} \\
\hline & $d=0$ & $d=1$ & $d=2$ & \\
\hline AT1530 & $2.73^{\mathrm{d}}$ & $1.91^{\mathrm{d}}$ & $-1.73^{\mathrm{d}}$ & 0.75 \\
\hline AVAT & 1.68 & 3.14 & -2.48 & 0.75 \\
\hline DAT1530 & 2.27 & 1.41 & -1.35 & 0.74 \\
\hline AVDAT & 1.48 & 3.52 & -2.44 & 0.75 \\
\hline NAT1530 & 3.01 & 2.13 & -1.84 & 0.75 \\
\hline AVNAT & 1.94 & 2.73 & -2.35 & 0.75 \\
\hline ARH90 & 10.41 & -1.78 & 1.22 & 0.77 \\
\hline AVARH & 11.66 & -2.66 & 1.32 & 0.78 \\
\hline DARH90 & 9.53 & -2.33 & 1.20 & 0.76 \\
\hline AVDARH & 11.43 & -2.73 & 1.42 & 0.77 \\
\hline NARH90 & 9.66 & -1.09 & 1.07 & 0.76 \\
\hline AVNARH & 10.96 & -2.20 & 1.05 & 0.78 \\
\hline DARH80 & 10.55 & -2.48 & 1.08 & 0.77 \\
\hline MINARH & 11.02 & -2.34 & 0.73 & 0.76 \\
\hline AT15RH90 & 10.11 & 1.03 & -0.29 & 0.77 \\
\hline DWD & 2.87 & -4.85 & 0.61 & \\
\hline TOTAL & 6.79 & -1.39 & 0.89 & 0.76 \\
\hline DURATION & 5.90 & -0.08 & 0.67 & 0.74 \\
\hline INTENSITY & 7.92 & -0.28 & 0.47 & 0.77 \\
\hline
\end{tabular}

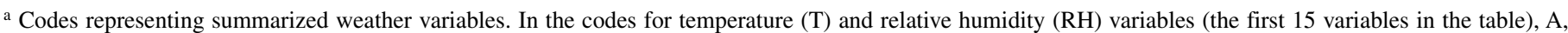
$\mathrm{D}, \mathrm{N}, \mathrm{AV}$, and MIN represent air, daytime, nighttime, average, and minimum, respectively. The numbers 15 and 1530 in the codes for temperature variables represent temperatures $>15$ and between 15 and $30^{\circ} \mathrm{C}$, respectively, whereas the numbers 80 and 90 in codes for $\mathrm{RH}$ variables represent $\mathrm{RH}>80$ and $>90 \%$, respectively. Variables for daytime periods represent summaries of conditions between 600 and $1800 \mathrm{~h}$, whereas variables for nighttime periods represent summaries of conditions between 1800 and $600 \mathrm{~h}$. With the exception of averages and minimum, which are given in ${ }^{\circ} \mathrm{C}$ or $\%$, all temperature and $\mathrm{RH}$ variables are given in terms of number of hours. So, for example, the codes DAT 1530 and AVNARH mean daytime air temperature between 15 and $30^{\circ} \mathrm{C}$ and average nighttime air relative humidity, respectively. DWD = daily wetness duration $(\mathrm{h})$, TOTAL $=$ total daily rainfall $(\mathrm{mm}), \mathrm{DURATION}=\mathrm{daily}$ rainfall duration $(\mathrm{h})$, and INTENSITY $=$ daily rainfall intensity $(\mathrm{mm} / \mathrm{h})$.

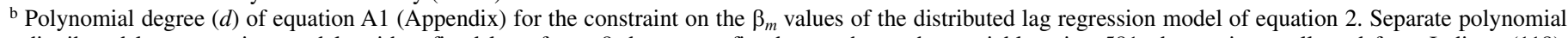
distributed lag regression models with a fixed lag of $n=8$ days were fitted to each weather variable using 581 observations collected from Indiana (118), Manitoba (21), North Dakota (115), South Dakota (108), Ohio (148), and Pennsylvania (71). Variables in this table were selected based on the magnitude of the mean square error and the Akaike information criterion.

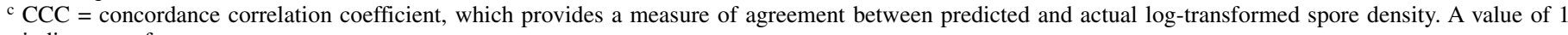
indicates perfect agreement.

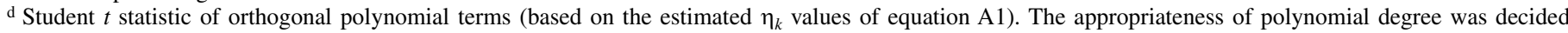
upon based on the absolute value of $t$, where the highest order term with the absolute value of $t>1.5$ was considered the appropriate polynomial degree (Appendix). 
PDL regression models only considered one weather variable at a time (although at several lags). Second, the mixed model accounted for the random effect of location-year and error structure (i.e., error structure) within location-years. Most of the estimated $\beta^{*}$ s were lower with the mixed models utilizing more than one weather variable than with the PDL models. In a loose sense, some of the predictive power of a weather variable was shared by
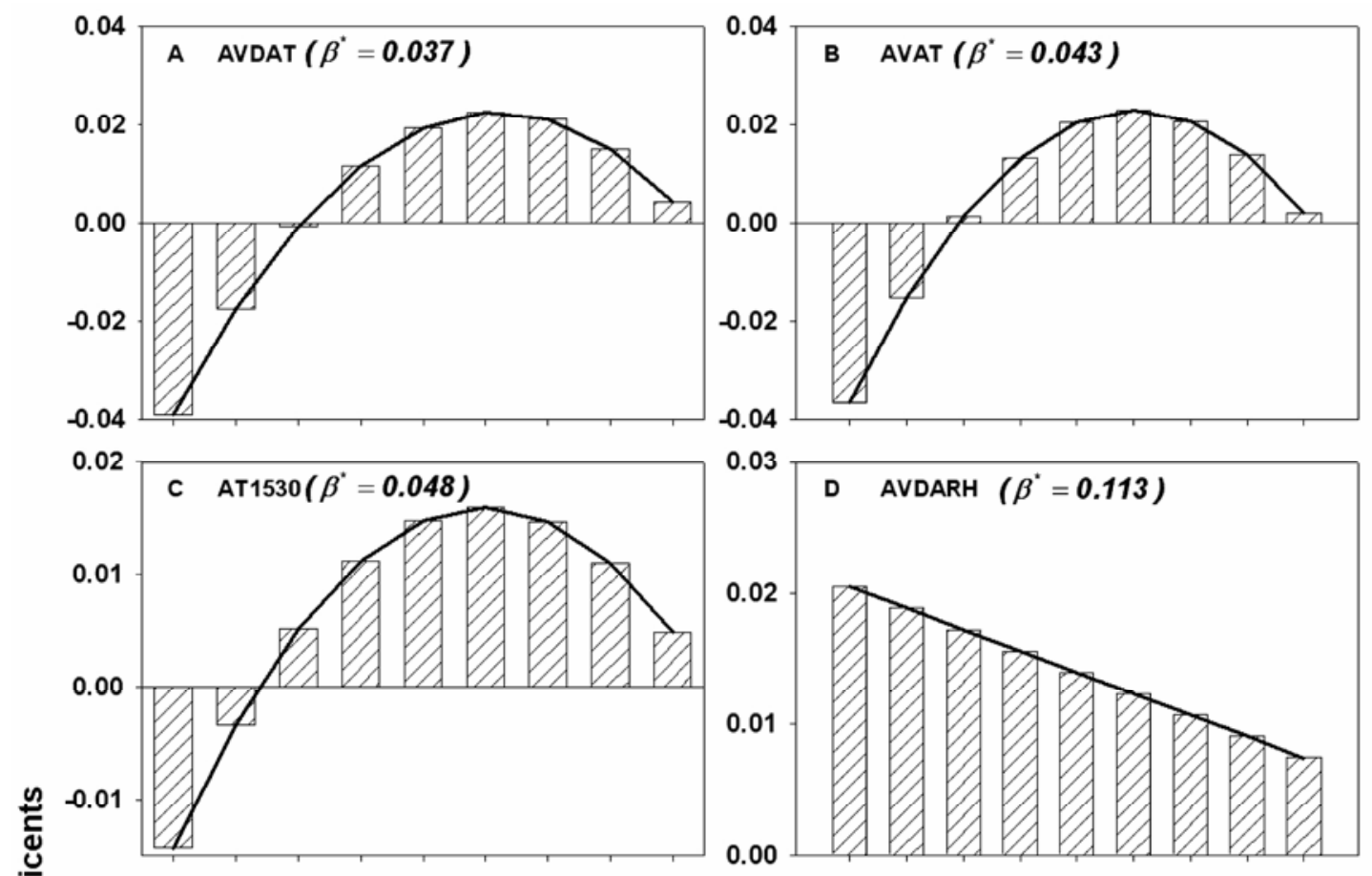
ocation-year effects.

Predictions of the mixed models. Figure 4 shows plots of actual $Y$ against predicted $Y(\hat{Y})$, along with the best fitting lines and the lines of perfect fit (the so-called concordance line; intercept $=0$ and slope $=1$ ) for the five models described in Table 4 (Fig. 4A, C, E, G, and I). In general, all plots showed
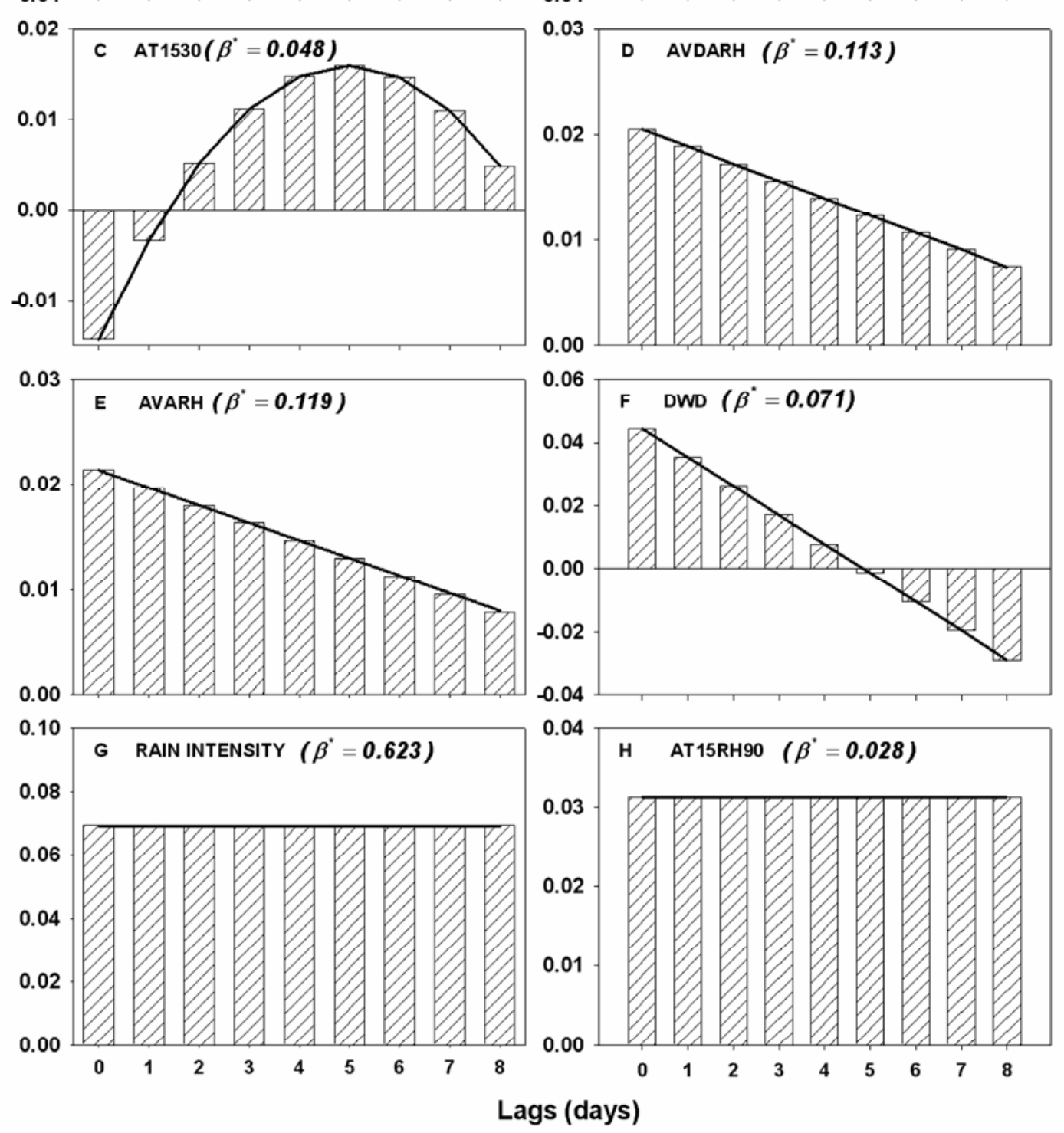

Fig. 3. Plots of estimated lag distributed parameters $\left(\hat{\beta}_{m}\right)$ from the fit of equation 2 (with a lag length of $n=8$ [m $\left.=0, \ldots, n\right]$ ) to log-transformed daily spore density of Gibberella zeae per wheat spike $(Y)$ for models based on A, average daytime (0600 to $1800 \mathrm{~h}$ ) air temperature, B, average daily air temperature, C, number of hours per day with air temperature between 15 and $30^{\circ} \mathbf{C}, \mathbf{D}$, average daytime relative humidity of the air, $\mathbf{E}$, average daily relative humidity of the air, $\mathbf{F}$, daily wetness duration, $\mathbf{G}$, rainfall intensity, and $\mathbf{H}$, number of hours per day with air temperature $>15^{\circ} \mathrm{C}$ and relative humidity $>90 \%$. Parameters were estimated by constraining the parameters to follow a polynomial curve (equation 2 and equations A1 to A3 in the Appendix) of order $d=0(\mathbf{G}, \mathbf{H}), d=1(\mathbf{D}, \mathbf{E}, \mathbf{F})$, or $d=2(\mathbf{A}, \mathbf{B}, \mathbf{C})$, based on the $t$ tests of the orthogonal polynomial terms shown in Table 3 for each weather variable. The $\beta^{*}$ value shown on each graph is the sum of the $\hat{\beta}_{m}$ values and represents the marginal effect of the weather variable on $Y$ over the 9 days. 
a very similar pattern that was consistent with a linear relationship between predicted and actual $Y$. The best-fit lines were all very close to the concordance lines, with all models slightly under-predicting at high $Y$ and slightly over-predicting at low $Y$ values. The concordance correlation coefficient (CCC) values were similar for all models $(\approx 0.80)$, suggesting that changing predictors from one model to another did not have a major effect on the overall prediction accuracy. There was little to discern
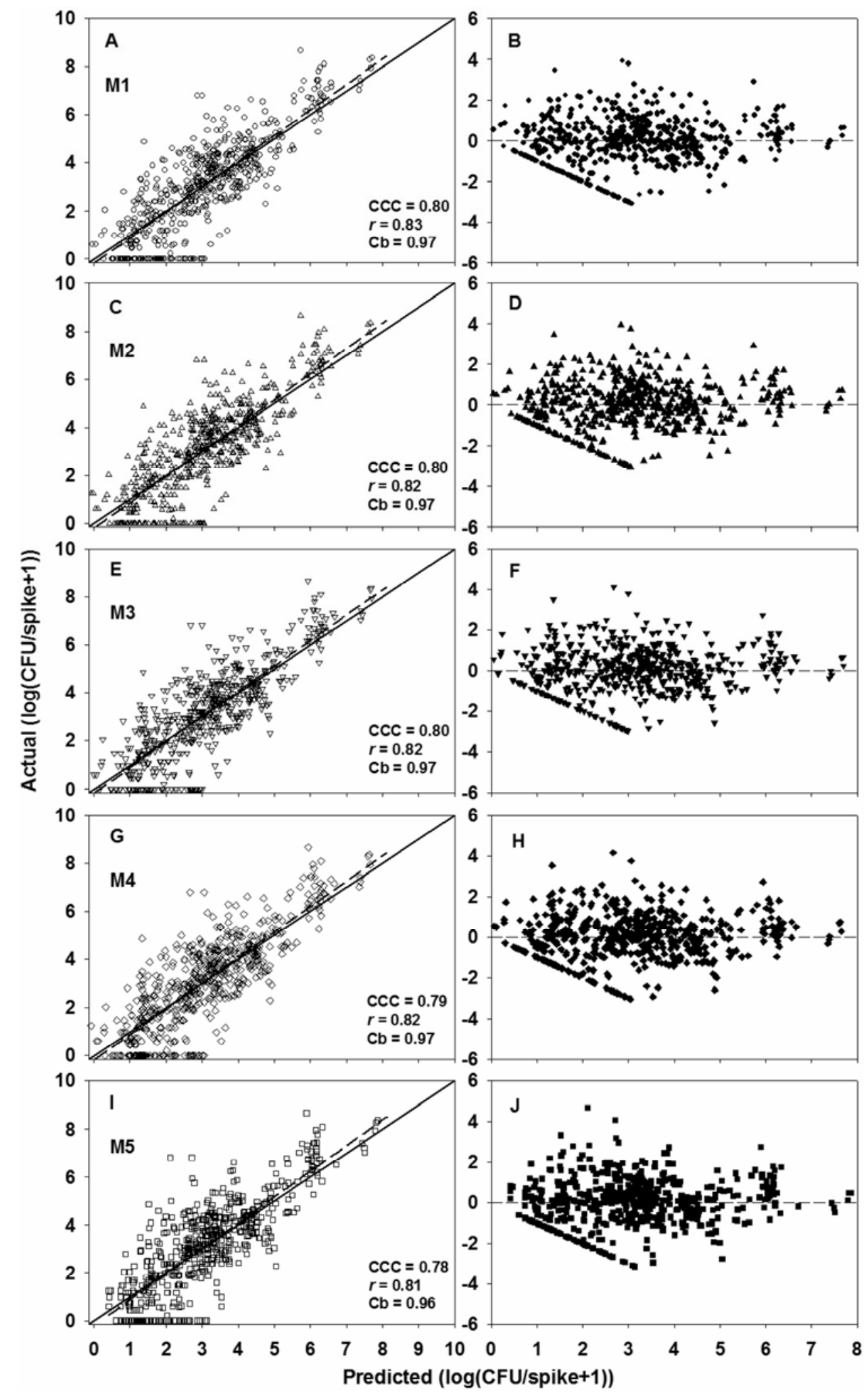

Fig. 4. Relationship between actual (observed) and predicted transformed daily spore density of Gibberella zeae on wheat spikes $[Y=\log (\mathrm{CFU} / \mathrm{spike}+1)](\mathbf{A}, \mathbf{C}$, $\mathbf{E}, \mathbf{G}$, and $\mathbf{I})$ and corresponding studentized residual plots $(\mathbf{B}, \mathbf{D}, \mathbf{F}, \mathbf{H}$, and $\mathbf{J})$ for five linear mixed models developed using different combinations of composite weather variables (Table 4). Solid and broken lines in $\mathbf{A}, \mathbf{C}, \mathbf{E}, \mathbf{G}$, and I represent perfect agreement between observed and predicted $Y$ (i.e., intercept $=0$ and slope $=1$; the "concordance line") and least-squares best-fit between observed and predicted $Y$, respectively. Inserts show the concordance correlation coefficient (CCC) (literature citations 32 and 34 [Chapter 2]), a measure of model accuracy, together with its two component terms, Pearson's product moment correlation coefficient $(r)$ and bias correction factor $\left(\mathrm{C}_{b}\right)$. CCC $=\mathrm{r} \cdot \mathrm{C}_{b} ; r$ quantifies the variability of the predictions (precision) and $\mathrm{C}_{b}$ quantifies the closeness of the best-fit line to the concordance line (bias). 
among the fits of these five models based on the graphs or fit statistics.

There was no discernable systematic pattern in the scatter of the studentized residuals for any of the models (Fig. 4B, D, F, H, and $\mathrm{J}$ ), an indication that the mixed models were reasonable for describing the data. The random pattern was an indication, in part, that the assumption of a linear relationship between $Y$ and the composite weather variables (as in equation 4) was justified, and that the random-effects terms accounted reasonably for the amonglocation year variability and the serial correlation of the residuals (52). The normal probability plots of the residuals (data not shown) indicated that the normality assumption for the data was reasonable for the analysis.

Predicted $Y$ from model M1 and actual $Y$ were plotted against time for each location (Fig. 5). In general, the model captured the day-to-day variation in $Y$, with predicted $Y$ followed a similar temporal pattern as observed $Y$ for all locations. The greatest discrepancies between actual and predicted were observed typically when actual $Y$ was equal to zero (Figs. 4 and 5). Moreover, the fluctuations in the predicted values often were of less magnitude than of the observed fluctuations within location-years; that is, the predictions typically did not reach the highest or the lowest values of $Y$.

Influence analysis. In general, the influence of data from individual location-years on the fit of the model and the stability of parameter estimates was similar for all the different mixed models considered in Table 4. Only results for model M1 are shown (Table 5). Overall, the fitted models were very robust in the sense that removal of the individual location-years had little effect on the results (Table 5). The log-likelihood distance $(l)$-which shows the fit of the model to all data when a given location-year was not used in estimating parameters-was small for all locations except for Indiana in 2001 (IN01). This was the only value above the critical threshold of 22 (calculated by PROC MIXED) for influential points based on the 75th percentile of the chisquare distribution (33). Cook's distance for the fixed-effect parameters $\left(D_{P}\right)$-which is a normalized metric showing how much the set of fixed-effect parameters change if a location-year is omitted-was also low for all location-years except for IN01 (0.37). Even this value was below the critical value of 1 based on the 50th percentile of the $F$ distribution. Cook's distance for the variance-covariance parameters $\left(D_{v}\right)$ was low for all locationyears except IN01. Thus, only one of the location-years was unusual in terms of the overall model fit, the estimates of the $\beta^{*}$ values, or the random-effect parameters (which determine the variances and covariances). With IN01 not used in the model fitting, the $\beta^{*}$ parameters for AVARH* ${ }^{*}$ AVDAT*, and INTENSITY* in model M1 would have been $0.086,0.010$, and 0.254 , respectively. Thus, the $\beta^{*}$ values would have been somewhat larger for AVARH* ${ }^{*}$ and smaller for AVDAT* ${ }^{*}$ and INTENSITY* (Table 4) if IN01 was not used. However, since the omission of IN01 did not provide substantial improvement in overall model performance, we opted to retain this location-year and use all of the available data for data analysis.

\section{DISCUSSION}

Utilizing a PDL regression analysis coupled with a linear mixed model analysis, we demonstrated that the log of daily inoculum density of $G$. zeae on wheat spikes $[Y=\ln (\mathrm{CFU} / \mathrm{spike}+1)]$ was statistically related to several weather conditions, not only on the day of sampling, but also on several days prior to sampling. The response to weather conditions was distributed over 9 days, including the current day, with many summary weather variables being associated with inoculum density on the spike. Nine days was the maximum time lag that could be evaluated, given the length of the within-location-year profiles of spore density and the available weather data. Relative humidity-related and wetness duration variables were the best predictors, although temperature and rainfall variables also were significantly related to spore density.

PDL models are novel in plant pathology, but they have been used successfully in several other disciplines for many decades, especially in econometrics $(1,7,42)$. In the last decade, the approach has been used fairly extensively in human epidemiology, especially to relate air pollution to daily mortality in cities in the United States $(8,55)$ and to relate weather and other conditions to infectious disease cases in East Africa and Australia (62). As discussed in the Materials and Methods, key advantages of this method are that it reduces (often drastically) the effect of the serial correlation of the predictor variables on the modeling results and it provides a formal statistical approach for selecting the temporal lag length $(n)$ and the structural relationship between the parameters and the temporal lag $\left(\beta_{m}\right.$ in relation to $\left.m\right)$. To determine effects of a variable distributed over several times, there must be a large number of observations $(>80)$ used in the model

TABLE 4. Estimated parameters ${ }^{\mathrm{a}}$ and corresponding standard errors (in parentheses) of five mixed models (equation 4) for the effects of composite weather variables and location on transformed daily spore density of Gibberella zeae on wheat spikes $[Y=\log (\mathrm{CFU} / \mathrm{spike}+1)]$

\begin{tabular}{|c|c|c|c|c|c|}
\hline \multirow[b]{2}{*}{ Term $^{\text {b }}$} & \multicolumn{5}{|c|}{ Models ${ }^{\mathrm{c}}$} \\
\hline & M1 & M2 & M3 & M4 & M5 \\
\hline AVARH $^{*}$ & $0.062(0.015)$ &.$^{d}$ & $0.064(0.015)$ & & . \\
\hline AVDARH* & $\ldots$ & $0.052(0.015)$ & $\ldots$ & $0.055(0.015)$ & $\ldots$ \\
\hline AT $1530^{*}$ & $\ldots$ & $\ldots$ & $0.030(0.010)$ & $0.031(0.011)$ & \\
\hline AT15RH90* & & & & & $0.097(0.041)$ \\
\hline INTENSITY* & $0.306(0.093)$ & $0.319(0.093)$ & $0.288(0.094)$ & $0.298(0.094)$ & $0.353(0.099)$ \\
\hline Ohio & $-2.971(1.213)$ & $-2.011(1.111)$ & $-3.257(1.217)$ & $-2.348(1.113)$ & $1.711(0.470)$ \\
\hline Pennsylvania & $-2.566(1.289)$ & $-1.572(1.185)$ & $-2.861(1.291)$ & $-1.917(1.184)$ & $2.127(0.618)$ \\
\hline South Dakota & $-0.230(1.224)$ & $0.673(1.137)$ & $-0.582(1.232)$ & $0.265(1.143)$ & $4.309(0.561)$ \\
\hline
\end{tabular}

a Estimates of the marginal effects (i.e., $\beta_{(1)}{ }^{*}, \beta_{(2)}{ }^{*}, \beta_{(3)}{ }^{*}$, or more generally, $\beta^{*}$ ) for composite weather variables and location effects in equation 4 . For location, each value shown represents the sum of the estimated $\alpha$ and individual location effect ( $\left.\hat{\alpha}+\hat{L}_{i}\right)$ of equation 4 . Thus, the value for each location is an overall intercept term representing predicted $Y$ when all composite weather variables are 0 .

${ }^{\mathrm{b}}$ Composites $\left(X^{*}\right.$; equation 3$)$ of average daily relative humidity of the air (AVARH), average daytime (600 to 1800 h) relative humidity of the air (AVDARH), average daytime air temperature (AVDAT), number of hours per day with air temperature between 15 and $30^{\circ} \mathrm{C}$ (AT1530) and rainfall intensity, and location category. The weights for the constructions of the weather composites in equation 3 were based on the polynomial distributed lag regression analysis (equation 2 ) done before the mixed model analysis.

${ }^{\mathrm{c}}$ Five models (M1 to M5) consisting of location and different combinations of composite weather variables developed using 581 observations from six locations.

d $\ldots=$ particular composite weather variable $\left(X^{*}\right)$ was not used in the model. 
fitting. Thus, the models could not be fitted individually for any single location-year, and would be debatable for a single location. However, in an approach similar to that taken by Teklehaimanot et al. (62), a fixed location effect was incorporated into the model (equation 2) to account for overall differences among locations in $\log$ spore density. Essentially, this means that each location had (potentially) a unique intercept term in the model (controlling the height of the curve of $Y$ versus time). As such, our approach identifies relationships between $Y$ and weather variables that hold across all location-years; the weather variables providing the best overall prediction accuracy of $Y$ may not necessarily be the best predictors for a single location or location-year (if such predictors could be precisely determined).

Results from the PDL regressions were highly consistent in terms of the appropriate lag length $(n=8$, the longest lag justifiable by the data) and the form of the relationship between $Y$ and the predictor variables. With PDL analysis, the form is defined by the degree of the polynomial $(d)$ that describes how $\beta_{m}$ changes with lag (from $m=0$ to $n$; Appendix), which can be viewed as the extremeness of the constraint placed on the $\beta_{m}$ parameters (the smaller the $d$, the larger the constraint). The largest significant $d$ was 2 (i.e., a quadratic function), which was found for several
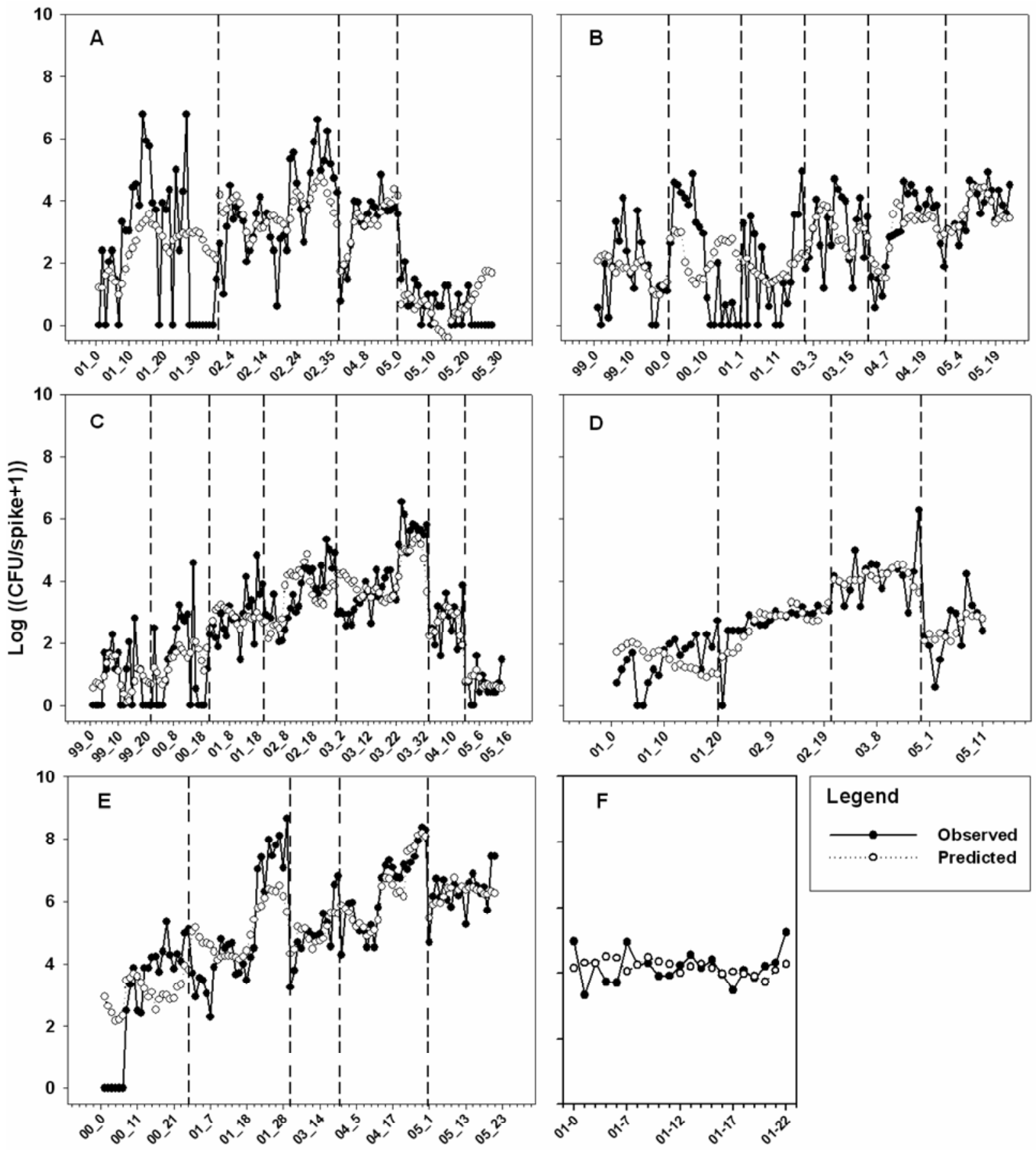

Year_Day

Fig. 5. Temporal variation in observed and predicted transformed daily spore density of Gibberella zeae on wheat spikes $[Y=\log (\mathrm{CFU} / \mathrm{spike}+1)]$ from the fit of linear mixed model M1 to the data (Table 4) for A, Indiana, B, North Dakota, C, Ohio, D, Pennsylvania, E, South Dakota, and F, Manitoba, Canada. Broken, vertical lines separate individual years within each location. Predictions of $Y$ were based on the estimated fixed-effects and random-effects terms in the model (equation 4). 
temperature-related variables. For the other variables, $d$ values of 1 or 0 were found. With these constraints, the $\beta_{m}$ values were either constant over time lag $(d=0$; rainfall variables and some temperature variables), declined monotonically with time lag ( $d=$ 1 ; most moisture-related variables), or increased to a maximum at an intermediate time lag (around 4 to 5 days) and then declined ( $d=2$; most temperature-related variables). The true relationship could be more complicated than this, but the data did not provide evidence to use more complicated polynomials to represent the $\beta_{m}$ values. In all cases, the marginal effect of a weather variable on $Y$ (i.e., $\beta^{*}=\Sigma \beta_{m}$ ) was positive, even when some individual $\beta_{m}$ estimates were negative.

To account for the between-location-year variability and the correlation (covariance) of observations within location-years, a linear mixed model analysis was conducted using composite weather variables ( $X^{*}$; equation 3 ) identified from the distributedlag regressions. In a more cumbersome procedure, a combined mixed-model PDL analysis could have been performed directly on the lagged weather variables instead of on the $X^{*}$ variables, using the general constraint approach of Edwards et al. (16). We found in a comparison of approaches, however, the same general results with the less cumbersome procedure described here (L. V.

TABLE 5. Statistics for evaluating the influence of individual location-years on the fit and estimated parameters of linear mixed model M1 (Table 4) for the relationship between transformed daily inoculum density of Gibberella zeae on wheat spikes $[Y=\log (\mathrm{CFU} /$ spike +1$)]$ and three composite weather variables $^{\mathrm{a}}$ and a location effect

\begin{tabular}{|c|c|c|c|}
\hline Location-year ${ }^{\mathrm{b}}$ & $l^{\mathrm{c}}$ & $D_{P}{ }^{\mathrm{d}}$ & $D_{v}{ }^{\mathrm{e}}$ \\
\hline IN01 & 32.50 & 0.37 & 16.40 \\
\hline IN02 & 4.24 & 0.10 & 2.67 \\
\hline IN04 & 0.24 & 0.01 & 0.16 \\
\hline IN05 & 1.43 & 0.13 & 0.24 \\
\hline MB01 & 0.26 & 0.02 & 0.18 \\
\hline ND99 & 0.64 & 0.04 & 0.24 \\
\hline ND00 & 1.30 & 0.03 & 0.92 \\
\hline ND01 & 0.81 & 0.02 & 0.60 \\
\hline ND03 & 0.35 & 0.01 & 0.21 \\
\hline ND04 & 0.59 & 0.04 & 0.22 \\
\hline ND05 & 0.57 & 0.04 & 0.17 \\
\hline OH99 & 0.32 & 0.02 & 0.13 \\
\hline $\mathrm{OH} 00$ & 0.89 & 0.02 & 0.67 \\
\hline OH01 & 0.15 & 0.00 & 0.12 \\
\hline $\mathrm{OH} 02$ & 0.82 & 0.06 & 0.32 \\
\hline $\mathrm{OH} 03$ & 1.03 & 0.06 & 0.50 \\
\hline OH04 & 0.22 & 0.01 & 0.12 \\
\hline OH05 & 0.33 & 0.03 & 0.09 \\
\hline PA01 & 0.71 & 0.06 & 0.15 \\
\hline PA02 & 0.22 & 0.00 & 0.20 \\
\hline PA03 & 0.86 & 0.07 & 0.17 \\
\hline PA05 & 0.12 & 0.00 & 0.08 \\
\hline SD00 & 2.02 & 0.13 & 0.55 \\
\hline SD01 & 0.55 & 0.03 & 0.32 \\
\hline SD03 & 0.13 & 0.00 & 0.10 \\
\hline SD04 & 0.74 & 0.06 & 0.21 \\
\hline SD05 & 0.31 & 0.02 & 0.12 \\
\hline
\end{tabular}

a Composite weather variables were: average daily relative humidity of the air (AVARH), average daytime air temperature (AVDAT), and rainfall intensity ( $X^{*}$; equation 3$)$.

b State and year from which data were collected to model the relationship between weather and Y. A total of 581 observations were collected from Indiana (IN), Manitoba (MB), North Dakota (ND), Ohio (OH), Pennsylvania (PA), and South Dakota (SD) from 1999 to 2005.

${ }^{c}$ Log-likelihood distance which provides an overall measure of the fit of the model when a given location-year was not used in estimating parameters. A value above a critical threshold of 22 indicates that a location-year is influential, based on the 75th percentile of the chi-square distribution.

d Cook's distance for fixed effect parameters which is a normalized metric showing how much the set of fixed-effect parameters change if a locationyear is omitted. A value above the critical value of 1 , based on the 50th percentile of the $F$ distribution, indicates that a location-year is influential.

e Cook's distance for variance-covariance, which is a normalized measure of the effect of each location-year on variability.
Madden, unpublished data). The mixed model analysis demonstrated that there was both significant between-location-year variability and a moving-average type of positive serial correlation within location-years (Toeplitz type error structure with order 7). The former indicates that unique (and unmeasured) environmental and biological factors in each location-year affected $Y$. The latter indicates that, conditional on the year-within-location random effect, $Y$ on a given day was positively associated with $Y$ on the 6 days prior to the given day. Such temporal autocorrelation is common with data collected over time $(34,52)$. Using the mixed model results, several possible two- or three-weather-variable models were identified with approximately equal prediction accuracy and interpretation.

The distributed lag relationship between weather and log-transformed $G$. zeae inoculum density on wheat spikes likely reflects the fact that the number of spores on the spike on any given day is a function of several interrelated processes of spore production and maturation, survival, and dissemination (liberation, transport, and deposition), all of which depend on periods of favorable weather conditions, which may occur over several days $(9,34,36)$. It is thus not possible to give a unique biological or epidemiological mechanism for the model results. However, previous studies suggest several (possibly conflicting or interacting) processes underlying the patterns of spore densities on wheat spikes in relation to weather within each location-year. G. zeae survives in crop residue left on the soil surface $(28,60)$, producing ascospores, macroconidia, and hyphal fragments, which may all serve as inoculum for FHB development. The production of perithecia, ascospores, and macroconidia is strongly influenced by temperature and moisture $(3,15,49,56,59,60)$, and several studies have demonstrated that the time taken for ascospore-producing (mature) perithecia to develop varies in response to these two weather variables $(15,63,66)$. Dufault et al. $(15)$ demonstrated that at a corn stalk water potential of $-0.45 \mathrm{MPa}$ and temperatures of 20 and $24^{\circ} \mathrm{C}$, mature perithecia of $G$. zeae were produced on corn stalks after an incubation period of 10 days, but not until 5 day later at $16^{\circ} \mathrm{C}$. Decreasing the water potential to $-1.30 \mathrm{MPa}$ led to a 5-day delay in perithecia maturation at $24^{\circ} \mathrm{C}$ and no observed maturation after 20 days at $16^{\circ} \mathrm{C}$. The 10 -day optimum time for perithecia maturation under continuous favorable conditions was consistent with observations made earlier by Tschanz et al. (66). However, contrary to what was reported by Dufault et al. (15), they found that the optimum temperature for ascospore production was $29^{\circ} \mathrm{C}(68)$, which was very similar to the optimum temperatures for macroconidia production $(2,49)$. In addition to perithecia maturation, water potential also influences the rate of perithecia production, with more perithecia being produced at higher compared with lower water potential $(15,59)$.

Once produced, spores must be passively released or actively discharged, transported, and deposited on wheat spikes $(21,36)$. Because weather may not always be conducive to all of these processes, it may take from a few hours to several days from the time spores are produced to the time they are deposited on the spike. Moreover, favorable conditions for one process may be unfavorable for another, potentially contributing to a time lapse between spore production and deposition or in obscuring any underlying relationships. Several studies have been conducted to evaluate the influence of rain and other weather variables on the discharge and dispersal of spores of $G$. zeae $(20,45,46,64,65,67)$. Due to differences in methodology and possibly inherent differences among the conditions under which the experiments were conducted, the results have been highly variable and sometimes conflicting. An important conclusion drawn from the results of most of these studies, however, is the fact that moisture (or lack there of) is considered to be a major limiting factor for spore dissemination $(45,64,65,67)$. This partly supports the consistent association observed in the current investigation between moisturerelated variables and inoculum density on wheat spikes. 
Tschanz et al. (67) demonstrated that the discharge of ascospores of $G$. zeae (based on the number of spores trapped above perithecia-bearing substrates under controlled conditions) was associated with cyclic wet and dry periods, suggesting that wet conditions were first needed for ascospore maturation followed by a period of substrate desiccation necessary for ascospore liberation. Paultiz (47) also observed an association between ascospore release and alternating wet and dry periods, with the daily maximum number of spores trapped over wheat plots inoculated with perithecia-bearing corn kernels occurring during periods of high relative humidity and surface wetness. Contrary to Tschanz et al. (67), Trail et al. $(64,65)$ reported that free water was needed for maximum ascospore discharge, and speculated that the association was probably due to the fact that free water fostered continuous ascospore maturation (and consequently, discharge), which did not occur under dry conditions. Parry et al. (43) suggested that discrepancies among reports of moisture effects on G. zeae spore dispersal were probably due to differential effects of different forms on moisture on initial ascospore release from perithecia, forcible ejection of ascospore, and the removal of macroconidia from the gelatinous matrix of sporodochia.

Wind may transport airborne spores to wheat spikes $(20,47,43)$. However, precipitation also plays a significant role in spore dispersal (45). Paulitz (47) observed that the concentration of ascospores of $G$. zeae in the air above inoculated wheat fields decreased following days of heavy rainfall, and suggested that this was probably because rain washed spores from the air. Similarly, based on the association between rainfall amounts and the numbers of propagules (CFU counts) of G. zeae collected in open petri plates at ear height in corn fields, Schmale and Bergstrom (54) reported that rainfall contributed to the deposition of airborne spores of this pathogen. Spores washed from the air during precipitation may either be deposited directly onto the spikes or intercepted by other plant parts from where they may then be splashed onto the spikes $(27,69)$. Paul et al. (45) observed that spores of $G$. zeae were consistently splash dispersed to spike height (approximately $100 \mathrm{~cm}$ above the inoculum source) within wheat canopies, and, like Parry et al (43), concluded that this was partly because spores initially splashed from residue on the soil surface to mid and upper leaves were subsequently resplashed to higher levels within the canopy. In the presence of free water on perithecia-bearing substrates and under conditions of high relative humidity, ascospores may ooze out of the perithecia instead of being forcibly discharged into the air $(47,65)$. Under such conditions, spores are typically splash dispersed (21).

As discussed above, temperature and moisture (including rainfall) play critical roles in determining the temporal processes occurring from spore production to deposition on the spikes. In an empirical sense, the estimated $\beta_{m}$ parameters of the PDL models capture the multiple temporal dynamic processes involved, including underlying interactions that could cancel out individual relationships between variables. Because under uncontrolled field conditions many processes may be occurring simultaneously, the $\beta_{m}$ parameters can only reflect the combined effect of a variable on $Y$, and a single weather variable may be of importance for several processes of significance. In the case of rainfall, some investigators have reported a temporal lag in the association between this variable and G. zeae daily inoculum density (both on spikes and in the air), documenting peaks in spore abundance between 1 and 4 days after rainfall $(23,26,47,48)$. Presumably, rainfall was providing moisture needed for production and maturation of macroconidia and ascospores, which resulted ultimately in more spores that were later disseminated in the canopy. However, rainfall can have an immediate impact on spore dissemination through splash dispersal of propagules (45). Much earlier rains (e.g., 5 to 8 days before the current day) may also provide moisture that would facilitate spore production. Thus, it is not necessarily surprising that the magnitude of rainfall effect on log spore density on wheat spikes was constant and positive $\left(\beta_{0}=\right.$ $\beta_{1}=\ldots=\beta_{8}>0$ ) over all 9 days.

The same general conclusion can be drawn regarding the combined effect of temperature and relative humidity over the 9-day time window, expressed as the number of hours of air temperature greater than $15^{\circ} \mathrm{C}$ and relative humidity $>90 \%$ (AT15RH90). The constant, positive weight given to AT15RH90 over all lags was indicative of an interaction effect of temperature and relative humidity on spore production and dissemination. Such an interaction has been well documented for many biological processes, including G. zeae spore production $(15,60)$ and liberation $(4,47)$, where the relative effect of temperature is often influenced by relative humidity/moisture. It is possible that a sub-optimal temperature for a given process on a given day may have been compensated for by optimum relative humidity or vise versa, leading to a joint (combined) effect that was positive and constant over the 9 days.

While the overall positive marginal effects of temperature and relative humidity $\left(\beta^{*}\right)$ on spore density indicated that increases in temperature and relative humidity were associated with increases in spore density on the spikes, the magnitude of the daily effects of these variables varied during the time window. Air temperature had a significant negative effect close to the time that spikes were sampled and a significant positive effect between 3 and 7 days, with a maximum positive effect at a 5-day lag. Although relatively high temperatures (above $30^{\circ} \mathrm{C}$ ) have been reported to inhibit G. zeae spore production (56), this was probably not the reason for negative $\beta_{m}$ parameters at low $m$, since the marginal effect of all temperature variables $\left(\beta^{*}\right)$ was positive in this study (i.e., a unit increase in the weather variable always resulted in the long term in an increase in $Y$ ). More likely, the quadratic relationship between the $\beta_{m}$ estimates and time lag reflected the time periods required for spore production and maturation, as discussed above. The immediate positive impact of relative humidity (e.g., $\beta_{0}, \beta_{1}$ ) on spore density, in contrast, likely reflects the fact that high relative humidity is often associated with peaks in spore liberation (47) and deposition (53) (based on airborne spore concentration), which probably occurs during days 1 and 0 of the lag window. Furthermore, correlations of the temperature and humidity weather variables may have contributed to the realized values of the $\beta \mathrm{s}$. For instance, high relative humidity and low temperatures are characteristic of nighttime, when there are major peaks in airborne concentration of $G$. zeae inoculum $(4,26,47,54)$. The inverse relation between temperature and relative humidity could be partly responsible for the negative $\beta_{0}$ found for some temperature variables.

The linear mixed models incorporating the composite weather variables $\left(X^{*}\right.$; e.g., AVARH $\left.^{*}\right)$ identified with PDL regression generally captured the day-to-day variation in inoculum density on wheat spikes, with predicted density following a temporal pattern similar to observed density. More than one combination of composite weather variables in the model resulted in very similar predictions. Residual plots gave no evidence of problems with the model fit or inappropriateness of the assumptions underlying the mixed model (52). Based on the influence statistics, with one exception (IN01), model results were robust in that individual location-years had little discernable impact on the model fit or on the parameter estimates (for the variables selected to predict $Y$ across all location-years). There were no obvious features of the weather variables for IN01 (P. A. Paul and L. V. Madden unpublished data) that would indicate unusual predictor-variable values; however, the profile of $Y$ versus time was characterized by many very low values $(\sim 0)$ and two very large peaks in CFUs, with few values in between (Fig. 2). The greatest discrepancies between predicted and actual log-transformed spore density across all location-years occurred at extreme values of observed density, which could have been due, at least in part, to errors made during sampling of spikes and enumeration of propagules of G. zeae. 
Based on what is known about the distribution of diseased wheat spikes $(13,17)$ and the spatial pattern of viable propagules of $G$. zeae in wheat fields (53), heterogeneity in spore density across a plot is expected. That is, for any given location-year, under a similar set of weather conditions, considerable variation in levels of spike inoculum density may occur among samples from different sites within the same plot. By basing the daily results on means of multiple replicate samples, the effects of the heterogeneity would be reduced, but the effects cannot be eliminated. With high variability of count data, it is especially difficult to estimate means close to zero precisely (34).

Driven by a small set of weather variables, the mixed models developed in this study had an accuracy of about 0.8 , as defined by the CCC. Several models-involving a combination of composite moisture, temperature, and rainfall variables - gave similar predictions, and there was little to separate the models in this regard. Although the calculated accuracy can be considered high (32), there is room for considerable improvement in accuracy. Use of different weather variables for different locations could improve predictions because the set of variables utilized here were selected to provide the overall best fit and not the best fit for one location (at the expense of the fit for another location). However, identification of weather variables within a subset of the total dataset will be severely limited by the short time periods involved in each location-year and the smaller total number of observations available for analysis. The analysis done here was performed to determine relationships that transcend particular locations, rather than to determine the most accurate predictions in a particular location. Given that the summary weather variables considered in this study encompass a wide range of ways of representing the ambient microclimate, it is unlikely that there are missing weather variables to utilize. A major limitation on improving predictions based on local weather information is the fact that some G. zeae spores in a wheat canopy may originate distantly (at possibly many different and undefined locations) rather than in and around the canopy $(23,53)$. Since the point of origin of these spores would not be known, the weather conditions where those spores are produced or disseminated would also not be known. Nevertheless, significant relationships between several variables based on local weather and $Y$ were found, clearly showing that in addition to the effects of locations and years within locations on $Y$, daily variation in spore density was related to local weather variables.

\section{APPENDIX}

There are several possible ways of constraining the $\beta \mathrm{s}$ in distributed lag models (such as equation 1a) in order to reduce the effects of correlations of the predictor variables and obtain more stable results. One useful approach is to express the constrained $\beta s$ with the following polynomial expression:

$$
\beta_{m}=\sum_{k=0}^{d} \eta_{k} m^{k}
$$

where $d$ is the degree of the polynomial, $m$ is the lag index ( $m=0$, $\ldots, n)$ for the lagged predictor variables of equation 1a, and the $\eta_{k}$ values $(k=0, \ldots, d)$ are the (new) parameters of the model. Use of equation A1 with equation 1a produces a so-called PDL model $(1,7,55,67)$. If we assume that $d=2$ (a quadratic case), as an example, then there are three $\eta$ parameters, $\eta_{0}, \eta_{1}$, and $\eta_{2}$. Based on these $\eta$ s and the use of equation $A 1$, the $\beta$ s can be defined; for example, the first four parameters of a model with $n=8$ are given as $\beta_{0}=\eta_{0}, \beta_{1}=\eta_{0}+\eta_{1}+\eta_{2}, \beta_{2}=\eta_{0}+2 \eta_{1}+4 \eta_{2}$, and $\beta_{3}=\eta_{0}+$ $3 \eta_{1}+9 \eta_{2}$. Substituting the values from equation A1 into equation $1 \mathrm{~b}$, the (constrained) PDL model (with $d=2$ ) is written as

$$
\begin{aligned}
Y_{t}= & \alpha+\eta_{0} X_{t}+\left(\eta_{0}+\eta_{1}+\eta_{2}\right) X_{t-1}+\left(\eta_{0}+2 \eta_{1}+4 \eta_{2}\right) X_{t-2}+ \\
& \cdots+\left(\eta_{0}+n \eta_{1}+n^{2} \eta_{2}\right) X_{t-n}+e_{t}
\end{aligned}
$$

Algebraic rearrangement of equation A2 results in

$$
\begin{gathered}
Y_{t}=\alpha+\eta_{0}\left(X_{t}+X_{t-1}+X_{t-2}+\cdots+X_{t-n}\right)+ \\
\eta_{1}\left(X_{t-1}+2 X_{t-2}+\cdots+n X_{t-n}\right)+ \\
\eta_{2}\left(X_{t-1}+4 X_{t-2}+\cdots+n^{2} X_{t-n}\right)+e_{t}
\end{gathered}
$$

The linear combinations of the $\mathrm{X}$ values within parentheses do not involve unknown parameters. Thus, by constraining $\beta_{m}$ values to lie on a polynomial curve, the number of parameters to be estimated for each weather variable is reduced from the number of lags +2 (in our example with $n=8$, there are 10 parameters: $\beta_{0}$ through $\beta_{8}, \alpha$ ) down to $d+2$ (e.g., $\eta_{0}, \eta_{1}$, and $\eta_{2}, \alpha$ ). Interestingly, an unconstrained model is the same as a PDL model with polynomial degree equal to the temporal lag $(d=n)$. In fitting models to data, the actual estimated values of the $\eta$ s are of little interest in terms of interpretation. Rather, estimates of the $\beta \mathrm{s}$ are obtained by substituting estimates of the $\eta \mathrm{s}$ in equation 2 .

The model of equation 1a, with equation A1 for the constraints, can be expanded to accommodate more than one lagged predictor variable as well as multiple continuous and category variables that do not involve temporal lags $(7,55)$.

In model fitting, there are several ways of determining the degree of the polynomial (d) (7). In the PDLREG procedure of SAS, orthogonal polynomials (18) are constructed from the polynomial coefficients (the $\eta$ parameters of equation A1) when equation 2 is fitted to data. Because, by definition, the orthogonal polynomial terms are independent, they provide the basis for sequentially testing the degree of the polynomial for $\beta$. With a lag of $n$, a PDL model with $d=n$ is fitted to the data. Each of the estimated orthogonal polynomial terms is tested for equality to 0 using Student $t$ tests. The highest order term that is significantly different from 0 is an indication of the polynomial degree. For example, with $d=n=4$, if the $0,1,2$, and 3 powers are significant and the 4th-order power is not significant, then this indicates that a cubic polynomial $(d=3)$ is appropriate. For this example, the model can then be refitted with $n=4$ and $d=3$, or options can be specified in advance in the procedure to recover information on the fit of this (or other) constrained model with one run of the program. It should be noted that if the chosen $d$ is too low (relative to the true value), then the estimated $\beta$ s will be biased (7). Thus, a liberal value for the critical Student $t$ statistic can be used to help ensure that the selected polynomial degree is not unreasonably low. For instance, a polynomial term can be considered significant (and the term retained) if the absolute value of the Student $t$ statistic is greater than 1.5, corresponding to an achieved significance level of $P \cong 0.14$.

\section{ACKNOWLEDGMENTS}

Research support provided by the United States Wheat and Barley Scab Initiative. Salaries and additional research support for P. A. Paul and L. V. Madden were provided by state and federal funds to the Ohio Agricultural Research and Development Center, The Ohio State University. We thank W. Bardall and A. Johnston for preparing and maintaining field plots in Ohio, and S. El-Allaf for collecting spike samples during the initial stages of this project.

\section{LITERATURE CITED}

1. Almon, S. 1965. The distributed lag between capital appropriations and expenditures. Econometrica 33:178-196.

2. Andersen, A. L. 1948. The development of Gibberella zeae head blight of wheat. Phytopathology 38:595-611.

3. Andries, C., Jarosz, A., and Trail, F. 2000. Effects of rainfall and temperature on the production of perithecia by Gibberella zeae in field debris in Michigan. Pages 118-122 in: Proc. 2000 Natl. Fusarium Head Blight Forum, Michigan State University, East Lansing.

4. Ayers, J. E., Pennypacker, S. P., Nelson, P. E., and Pennypacker, B. W. 1975. Environmental factors associated with airborne ascospores of Gibberella zeae in corn and wheat fields. Phytopathology 65:835. 
5. Bai, G. 1995. Scab of wheat: Epidemiology, inheritance of resistance, and molecular markers linked to cultivar resistance. Ph.D. diss. Purdue University, West Lafayette, IN.

6. Bai, G., and Shaner, G. 1994. Scab of wheat: Prospects for control. Plant Dis. 78:760-766.

7. Batten, D. S., and Thornton, D. L. 1983. Polynomial distributed lags and the estimation of the St. Louis equation. Fed. Reserve Bank St. Louis Rev. April:13-25.

8. Braga, A. L. F., Zanobetti, A., and Schwartz, J. 2001. The time course of weather-related deaths. Epidemiology 12:662-667.

9. Campbell, C. L., and Madden, L. V. 1990. Introduction to Plant Disease Epidemiology. John Wiley \& Sons, New York.

10. Champeil, A., Dore, T., and Fourbet, J. F. 2004. Fusarium head blight: Epidemiological origin of the effects of cultural practices on head blight attacks and the production of mycotoxins by Fusarium in wheat grains. Plant Sci. 166:1389-1415.

11. Coakley, S. M., McDaniel, L. R., and Shaner, G. 1985. Model for predicting severity of Septoria tritici blotch on winter wheat. Phytopathology 75:1245-1251.

12. De Wolf, E., Francl, L., Lipps, P., Madden, L., Osborne, L., and Jin, Y. 2000. Factors affecting the development of wheat Fusarium head blight. Pages 137-140 in: Proc. 2000 Natl. Fusarium Head Blight Forum, Michigan State University, East Lansing.

13. Del Ponte, E. M., Shah, D. A., and Bergstrom, G. C. 2003. Spatial patterns of Fusarium head blight in New York wheat fields suggest role of airborne inoculum. Online. Plant Health Progress doi:10.1094/PHP-20030418-01-RS

14. Dill-Macky, R., and Jones, R. K. 2000. The effect of previous crop residues and tillage on Fusarium head blight of wheat. Plant Dis. 84:7176.

15. Dufault, N. S., De Wolf, E. D., Lipps, P. E., and Madden, L. V. 2006. Role of temperature and moisture in the production and maturation of Gibberella zeae perithecia. Plant Dis. 90:637-644.

16. Edwards, L. J., Stewart, P. W., Muller, K. E., and Helms, R. W. 2001. Linear equality constraints in the general linear mixed model. Biometrics 57:1185-1190.

17. El-Allaf, S. M., Madden, L. V., and Lipps, P. E. 2001. Spatial pattern of scab incidence during Fusarium head blight epidemics on winter wheat in Ohio. Pages 118-122 in: Proc. 2001 National Fusarium Head Blight Forum, Michigan State University, East Lansing.

18. Emerson, P. L. 1968. Numerical construction of orthogonal polynomials from a general recurrence formula. Biometrics 24:695-701.

19. Fauzi, M. T., and Paulitz, T. C. 1994. The effect of plant growth regulators and nitrogen on Fusarium head blight of the spring wheat cultivar Max. Plant Dis. 78:289-292.

20. Fernando, W. G. D., Paulitz, T. C., Seaman, W. L., Dutilleul, P., and Miller, J. D. 1997. Head blight gradient caused by Gibberella zeae from area sources of inoculum in wheat field plots. Phytopathology 87:414421.

21. Fitt, B. D. L., McCartney, H. A., and Walklate, P. J. 1989. The role of rain in dispersal of pathogen inoculum. Annu. Rev. Phytopathol. 27:241-270.

22. Francl, L. J., Madden, L. V., Rowe, R. C., and Riedel, R. M. 1990. Correlation of growing season environmental variables and the effect of early dying on potato yield. Phytopathology 80:425-432.

23. Francl, L., Shaner, G., Bergstrom, G., Gilbert, J., Pedersen, W., DillMacky, R., Sweets, L., Corwin, B., Jin, Y., Gallenberg, D., and Wiersma, J. 1999. Daily inoculum levels of Gibberella zeae on wheat spikes. Plant Dis. 83:662-666.

24. Garrett, K. A., Madden, L. V., Hughes, G., and Pfender, W. F. 2004. New applications of statistical tools in plant pathology. Phytopathology 94:999-1003.

25. Hsiao, C. 2003. Analysis of Panel Data. Cambridge University Press, UK.

26. Inch, S., Fernando, W. G. D., and Gilbert, J. 2005. Seasonal and daily variation in the airborne concentration of Gibberella zeae (Schw.) Patch spores in Manitoba. Can. J. Plant Pathol. 27:357-363.

27. Jenkinson, P., and Parry, D. W. 1994. Splash dispersal of conidia of Fusarium culmorum and Fusarium avenaceum. Mycol. Res. 98:506510 .

28. Khonga, E. B., and Sutton, J. C. 1988. Inoculum production and survival of Gibberella zeae in maize and wheat residues. Can. J. Plant Pathol. 10:232-239.

29. Komada, H. 1975. Development of a selective medium for quantitative isolation of Fusarium oxysporum from natural soils. Rev. Plant Prot. Res. 8:114-125.

30. Kutner, M. H., Nachtsheim, C. J., Neter, J., and Li, W. 2005. Applied linear statistical models. McGraw-Hill/Irwin, New York.

31. Large, E. C. 1954. Growth stages in cereals. Plant Pathol. 3:128-129.

32. Lin, L., Hedayat, A. S., Sinha, B., and Yang, M. 2002. Statistical methods in assessing agreement: Models, issues, and tools. J. Am. Stat. Assoc. 97:257-270.
33. Littell, R. C., Milliken, G. A., Stroup, W. W., Wolfinger, R. D., and Schabenberger, O. 2006. SAS System for Mixed Models. SAS Institute, Cary, NC.

34. Madden, L. V., Hughes, G., and van den Bosch, F. 2007. The Study of Plant Disease Epidemics. American Phytopathological Society, St. Paul, MN.

35. Markell, S., and Francl, L. 2003. Fusarium head blight inoculum: Species prevalence and Gibberella zeae spore type. Plant Dis. 87:814-820.

36. McCartney, H. A. 1994. Pages 171-185 in: Ecology of Plant Pathogens. Spore dispersal: Environmental and biological factors. J. P. Blakeman and B. Williamson, eds. CAB International Publishing, Wallingford, UK.

37. McMullen, M., Jones, R., and Gallenburg, D. 1997. Scab of wheat and barley: A re-emerging disease of devastating impact. Plant Dis. 81:13401348 .

38. Nelson, P. E., Toussoun, T. A., and Marasas, W. F. O. 1983. Fusarium Species: An Illustrated Manual for Identification. The Pennsylvania State University, University Park, PA.

39. Nita, M., Ellis, M. A., and Madden, L. V. 2003. Reliability and accuracy of visual estimation of Phomopsis leaf blight of strawberry. Phytopathology 93:995-1005.

40. Osborne, L. E., and Stein, J. M. 2006. Temporal inoculum dynamics for Fusarium head blight of wheat and barley in South Dakota. (Abstr.) Phytopathology 96(suppl.):S88.

41. Osborne, L., Yin, Y., and Kohl, R. 2000. Fusarium head blight: Inoculum detection, disease progress, and environmental influence. Pages 163-168 in: Proc. 2000 Natl. Fusarium Head Blight Forum, Michigan State University, East Lansing.

42. Pagano, M., and Hartley, M. J. 1981. On fitting distributed lag models subject to polynomial restrictions. J. Econometrics 16:171-198.

43. Parry, D. W., Jenkinson, P., and McLeod, L. 1995. Fusarium ear blight (scab) in small grain cereals-A review. Plant Pathol. 44:207-238.

44. Paul, P. A., El-Allaf, S. M., Lipps, P. E., and Madden, L. V. 2003. Development of Fusarium head blight of winter wheat in Ohio as influenced by planting date, cultivar maturity, and inoculum level. Pages 156-159 in: Proc. 2003 Natl. Fusarium Head Blight Forum, Michigan State University, East Lansing.

45. Paul, P. A., El-Allaf, S. M., Lipps, P. E., and Madden, L. V. 2004. Rain splash dispersal of Gibberella zeae within wheat canopies in Ohio. Phytopathology 94:1342-1349.

46. Paul, P. A., El-Allaf, S. M., Lipps, P. E., and Madden, L. V. 2004. Relationship between the environment and the amount of Gibberella zeae inoculum recovered from wheat heads in Ohio. (Abstr.) Phytopathology 94(suppl.):S82.

47. Paulitz, T. C. 1996. Diurnal release of ascospores by Gibberella zeae in inoculated wheat plots. Plant Dis. 80:674-678.

48. Rossi, V., Languasco, L., Pattori, E., and Giosue, S. 2002. Dynamics of airborne Fusarium macroconidia in wheat fields naturally affected by head blight. J. Plant Pathol. 84:53-64.

49. Rossi, V., Pattori, E., Ravanetti, A., and Giosue, S. 2002. Effect of constant and fluctuating temperature regimes on sporulation of four fungi causing head blight of wheat. J. Plant Pathol. 84:95-105.

50. Schaafsma, A. W., Tamburic-Ilincic, L., and Hooker, D. C. 2005. Effect of previous crop, tillage, field size, adjacent crop, and sampling direction on airborne propagules of Gibberella zeae/Fusarium graminearum, Fusarium head blight severity, and deoxynivalenol accumulation in winter wheat. Can. J. Plant Pathol. 27:217-224.

51. Schaafsma, A. W., Tamburic-Ilinic, L., Miller, J. D., and Hooker, D. C. 2001. Agronomic considerations for reducing deoxynivalenol in wheat grain. Can. J. Plant Pathol. 23:279-285.

52. Schabenberger, O., and Pierce, F. J. 2002. Contemporary Statistical Models for the Plant and Soil Sciences. CRC Press, New York.

53. Schmale, D. G., Shah, D. A., and Bergstrom, G. C. 2005. Spatial patterns of viable spore deposition of Gibberella zeae in wheat fields. Phytopathology 95:472-479.

54. Schmale, D. G., and Bergstrom, G. C. 2004. Spore deposition of the ear rot pathogen, Gibberella zeae, inside corn canopies. Can. J. Plant Pathol. 26:591-595.

55. Schwartz, J. 2000. The distributed lag between air pollution and daily deaths. Epidemiology 11:320-326.

56. Shaner, G. 2003. Epidemiology of Fusarium head blight of small grain cereals in North America. Pages 84-119 in: Fusarium Head Blight of Wheat and Barley. K. J. Leonard and W. R. Bushnell, eds. American Phytopathological Society, St. Paul, MN.

57. Shaner, G., and Buechley, G. 2000. Sampling spores of Fusarium graminearum. Pages 182-186 in: Proc. 2000 Natl. Fusarium Head Blight Forum, Michigan State University, East Lansing.

58. Stein, J. M., and Osborne, L. E. 2006. A relationship between the number of Gibberella zeae propagules present during heading and deoxynivalenol (DON) content in wheat grain. (Abstr.) Phytopathology 96(suppl.): S110. 
59. Sung, J.-M., and Cook, R. J. 1981. Effect of water potential on reproduction and spore germination of Fusarium roseum "Graminearum, 'Culmorum,' and 'Avenaceum'. Phytopathology 71:499-504.

60. Sutton, J. C. 1982. Epidemiology of wheat head blight and maize ear rot caused by Fusarium graminearum. Can. J. Plant Pathol. 4:195-209.

61. Teich, A. H., and Nelson, K. 1984. Survey of Fusarium head blight and possible effects of cultural practices in wheat fields in Lambton county in 1983. Can. Plant Dis. Surv. 64:11-13.

62. Teklehaimanot, H. D., Lipsitch, M., Teklehaimanot, A., and Schwartz, J. 2004. Weather-based prediction of Plasmodium falciparum malaria in epidemic-prone regions of Ethiopia I. Patterns of lagged weather effects reflect biological mechanisms. Malaria J. 41:1-11.

63. Trail, F., and Common, R. 2000. Perithecial development by Gibberella zeae: A light microscopy study. Mycologia 92:130-138.

64. Trail, F., Gadoury, D., and Loranger, R. 1998. Environmental parameters of ascospore discharge in Gibberella zeae. Pages 11-12 in: Proc. 1998 Natl. Fusarium Head Blight Forum, Michigan State University, East Lansing.
65. Trail, F., Haixin, X., Loranger, R., and Gadoury, D. 2002. Physiological and environmental aspects of ascospore discharge in Gibberella zeae (anamorph Fusarium graminearum). Mycologia 94:181-189.

66. Tschanz, A. T., Horst, R. K., and Nelson, P. E. 1975. A substrate for uniform production of perithecia in Gibberella zeae. Mycologia 67:11011108.

67. Tschanz, A. T., Horst, R. K., and Nelson, P. E. 1975. Ecological aspects of ascospore discharge in Gibberella zeae. Phytopathology 65:597-599.

68. Tschanz, A. T., Horst, R. K., and Nelson, P. E. 1976. The effect of environment on sexual reproduction of Gibberella zeae. Mycologia 68:327340 .

69. Walklate, P. J., McCartney, H. A., and Fitt, B. D. L. 1989. Vertical dispersal of plant pathogens by splashing. Part II: Experimental study of the relationship between raindrop size and the maximum splash height. Plant Pathol. 38:64-70.

70. Xu, X. 2003. Effect of environmental conditions on the development of Fusarium ear blight. Eur. J. Plant Pathol. 109:683-689. 